JOURNAL OF THE

AMERICAN MATHEMATICAL SOCIETY

Volume 22, Number 2, April 2009, Pages 569-605

S $0894-0347(08) 00622-\mathrm{X}$

Article electronically published on November 6, 2008

\title{
UNIFORM ENERGY DISTRIBUTION FOR AN ISOPERIMETRIC PROBLEM WITH LONG-RANGE INTERACTIONS
}

\author{
GIOVANNI ALBERTI, RUSTUM CHOKSI, AND FELIX OTTO
}

\section{INTRODUCTION}

This paper concerns the structure of minimizers for the following nonlocal variational (isoperimetric) problem in $\mathbb{R}^{n}$. For $m \in(-1,1)$ and $Q_{L}:=\left(-\frac{L}{2}, \frac{L}{2}\right)^{n} \subset \mathbb{R}^{n}$ :

$$
\text { Minimize } S(u)+\int_{Q_{L}} \int_{Q_{L}} G(x, y)(u(x)-m)(u(y)-m) d x d y
$$

where $u$ denotes a function defined on $Q_{L}$ taking the values 1 or -1 with fixed volume fractions so that

$$
\frac{1}{L^{n}} \int_{Q_{L}} u d x=m
$$

$S(u)$ denotes the interfacial area associated with the surfaces upon which $u$ jumps, and $G(x, y)$ denotes the Green's function for $-\triangle$ on $Q_{L}$ with Neumann boundary conditions. The variational problem consists of competing short-range $(S(u))$ and long-range (the nonlocal Green's function term) contributions. The former term is attractive, favoring large domains of pure phases with boundaries of minimal surface area. The latter term is repulsive, favoring small domains which lead to cancellations. The combination of the two leads to pattern formation on a scale determined solely by the competition of the two terms.

Mathematically, the natural space for $u$ is $B V\left(Q_{L}, \pm 1\right)$, functions of bounded variation taking values \pm 1 . The interfacial area is then simply half the total variation measure $|\nabla u|$ on $Q_{L}$, i.e.,

$$
S(u)=\frac{1}{2} \int_{Q_{L}}|\nabla u|
$$

With this notation, our problem can be restated as follows.

Received by the editors October 16, 2007.

2000 Mathematics Subject Classification. Primary 49Q10, 49N60, 49S05, 35B10.

(C)2008 American Mathematical Society Reverts to public domain 28 years from publication 
For $m \in(-1,1)$ and $Q_{L}=\left(-\frac{L}{2}, \frac{L}{2}\right)^{n} \subset \mathbb{R}^{n}$ :

$$
\text { Minimize } \int_{Q_{L}}|\nabla u|+\frac{1}{2} \int_{Q_{L}} \int_{Q_{L}} G(x, y)(u(x)-m)(u(y)-m) d x d y
$$

over all $u \in B V\left(Q_{L}, \pm 1\right)$ satisfying the mass constraint (1.1).

Problem (1.2) is the sharp interface limit (in the sense of Gamma-convergence) of a diffuse interface variational problem first introduced by Ohta and Kawasaki in 21] to model microphase separation in diblock copolymers (cf. 2]). Following [19, 9, this diffuse interface problem (suitably rescaled) can be written as follows: For $\epsilon$ small, minimize

$$
\int_{Q_{L}} \epsilon|\nabla u|^{2}+\frac{\left(1-u^{2}\right)^{2}}{4 \epsilon} d x+\int_{Q_{L}} \int_{Q_{L}} G(x, y)(u(x)-m)(u(y)-m) d x d y
$$

over all $u \in H^{1}\left(Q_{L},[-1,1]\right)$ satisfying (1.1). A derivation of (1.3) from the statistical physics of interacting block copolymers can be found in 9 . However, problem (1.2) (or (1.3) ) can be viewed as a mathematical paradigm for the ubiquitous phenomenon of energy-driven pattern formation induced by competing short- and longrange interactions. The connection between this type of energetic competition and periodic pattern formation in nature is well-established (see for example [25, 14, 18, and the references therein). The highly-cited article of Seul and Andelman 25. addresses exactly this issue and provides a partial list of relevant physical systems in Table 1. Problem (1.2) is also very close to variational problems arising in the study of magnetic domains and walls (see for example [15, 12] and the references therein).

In one space dimension, it can be proven that minimizers of either (1.2) or (1.3) are periodic ( $c f . \quad 1,17,22,4,29$ ). In fact, in one space dimension, a vanishing first variation of (1.2) is sufficient to enforce periodicity. Of course one-dimensional pattern formation is rather restrictive, and a fundamental mathematical problem is to address to what extent periodicity holds in higher space dimensions and what effect the nonlocal term has on the geometry of the interfaces which, in general, will not have constant mean curvature.

As we have mentioned, problem (1.2) is the sharp interface version of (1.3), which was derived to model microphase separation of diblock copolymers. There is a wealth of experimental literature on phase structures in diblock copolymer melts, where observations yield phase boundaries strongly resembling triply periodic constant mean curvature surfaces (see for example 2, 28, and the references therein). Moreover, numerical simulations on the diffuse interface functional concur with the experimental observations (see for example [26, 8]). Thus it is certainly natural to conjecture that in higher dimensions, minimizers are at least nearly periodic. However proving any periodicity result turns out to be a formidable task. In fact, even in a discrete setting with a pair interaction potential, proving periodicity is extremely difficult and was only recently successfully accomplished under certain assumptions in 27. In this article, we address a weaker statement, that of the spatial distribution of the energy density of minimizers. 
The rigorous derivation of the first and second variations of (1.2) was presented in [10] (with a formal calculation presented in the appendix of [17]). These methods are inherently local and pertain to local minimizers. While it is certainly possible that, for example, stability alone could enforce some sort of periodicity, here we apply direct methods which are appropriate for the analysis of global minimizers. To this end, we introduce a reformulation of the nonlocal term which, for our purposes, is most natural and proves crucial in the analysis.

Given $u \in B V\left(Q_{L}, \pm 1\right)$ with $\frac{1}{L^{n}} \int_{Q_{L}} u d x=m$, we have

$$
\begin{aligned}
& \int_{Q_{L}} \int_{Q_{L}} G(x, y)(u(x)-m)(u(y)-m) d x d y \\
& =\min \left\{\int_{Q_{L}}|\mathbf{b}|^{2} d x \mid \mathbf{b} \in L^{2}\left(Q_{L}, \mathbb{R}^{n}\right),\right. \\
& \left.\operatorname{div} \mathbf{b}=u-m \text { in } Q_{L}, \mathbf{b} \cdot \nu=0 \text { on } \partial Q_{L}\right\},
\end{aligned}
$$

where the differential equation relating $\mathbf{b}$ to $u$ and the Neumann boundary condition are interpreted in the sense of distributions. While it is rather straightforward to establish (1.4), we provide a proof for the convenience of the reader. Let $v$ be the solution of

$$
\left\{\begin{aligned}
-\Delta v=u-m & \text { in } Q_{L} \\
\nabla v \cdot \nu=0 & \text { on } \partial Q_{L}
\end{aligned}\right.
$$

Thus $v$ is unique up to addition of a constant and will often be denoted in the following as the potential associated to $u$. We claim that the minimum at the righthand side of (1.4) is attained when $\mathbf{b}=-\nabla v$, and it is equal to the left-hand side. The vector field $-\nabla v$ is indeed an admissible choice for $\mathbf{b}$, and since $v$ is given by

$$
v(x)=\int_{Q_{L}} G(x, y)(u(x)-m) d x
$$

an integration by parts yields

$$
\int_{Q_{L}} \int_{Q_{L}} G(x, y)(u(x)-m)(u(y)-m) d x d y=\int_{Q_{L}}(u-m) v d x=\int_{Q_{L}}|\nabla v|^{2} d x
$$

It remains to show that if $\mathbf{b} \in L^{2}\left(Q_{L}, \mathbb{R}^{n}\right)$ satisfies

$$
\operatorname{div} \mathbf{b}=u-m \text { in } Q_{L} \quad \text { and } \quad \mathbf{b} \cdot \nu=0 \text { on } \partial Q_{L},
$$

then we have

$$
\int_{Q_{L}}|\mathbf{b}|^{2} d x \geq \int_{Q_{L}}|\nabla v|^{2} d x
$$


To this end, note that

$$
\begin{aligned}
\int_{Q_{L}}|\mathbf{b}|^{2} d x & =\int_{Q_{L}}|\nabla v|^{2} d x+\int_{Q_{L}}|\mathbf{b}+\nabla v|^{2} d x-2 \int_{Q_{L}}(\mathbf{b}+\nabla v) \cdot \nabla v d x \\
& \geq \int_{Q_{L}}|\nabla v|^{2} d x+2 \int_{Q_{L}} \operatorname{div}(\mathbf{b}+\nabla v) v d x-2 \int_{\partial Q_{L}} v(\mathbf{b}+\nabla v) \cdot \nu d x \\
& =\int_{Q_{L}}|\nabla v|^{2} d x .
\end{aligned}
$$

We arrive at the following reformulation of problem (1.2):

$$
\text { Minimize } E\left(u, \mathbf{b}, Q_{L}\right) \text { over }(u, \mathbf{b}) \in \mathcal{A}_{0}\left(Q_{L}\right),
$$

where

$$
E\left(u, \mathbf{b}, Q_{L}\right):=\int_{Q_{L}}|\nabla u|+\frac{1}{2} \int_{Q_{L}}|\mathbf{b}|^{2} d x
$$

and

$$
\begin{aligned}
\mathcal{A}_{0}\left(Q_{L}\right):=\left\{(u, \mathbf{b}) \mid u \in B V\left(Q_{L}, \pm 1\right), \mathbf{b} \in L^{2}\left(Q_{L}, \mathbb{R}^{n}\right),\right. & \\
& \left.\operatorname{div} \mathbf{b}=u-m, \mathbf{b} \cdot \nu=0 \text { on } \partial Q_{L}\right\} .
\end{aligned}
$$

This reformulation has several advantages. For example, it localizes the functional and facilitates the use of cutting and pasting arguments. It further allows us to demonstrate that the particular choice of boundary conditions does not influence the structure away from the boundary - our main result will support this. In fact, in this reformulation, one could even dispense entirely with the boundary conditions for $\mathbf{b} \cdot \nu$; in that case, the correct mean (cf. (1.1) $)$ is enforced as a soft constraint rather than a hard constraint.

Note that using the direct method in the calculus of variations, it is straightforward to show that a minimizer of (1.7) exists. Our first two results concern the uniform distribution of its energy. We prove that for a minimizer on a large cube $L \gg 1$, if we zoom in on any subcube of size $l \gg 1$, we find essentially the same amount of energy. Let us state this precisely. For any $l>0$, let $Q_{l}(a)$ denote the cube in $\mathbb{R}^{n}$ centered at $a$ with side $l$, i.e.,

$$
Q_{l}(a):=a+\left(-\frac{l}{2}, \frac{l}{2}\right)^{n},
$$

with the understanding that if the argument $a$ is absent, we take $a=0$.

Theorem 1.1. There exist positive constants $\sigma^{*}, C, c$, depending only on the volume fraction $m$ and space dimension $n$, with the following property: If $\left(u_{0}, \mathbf{b}_{0}\right)$ is a minimizer of $E\left(u, \mathbf{b}, Q_{L}\right)$ on $\mathcal{A}_{0}\left(Q_{L}\right)$, then for every $l \geq c$ and $a \in Q_{L}$ such that $Q_{l}(a) \subset Q_{L}$, we have

$$
\left|\frac{E\left(u_{0}, \mathbf{b}_{0}, Q_{l}(a)\right)}{l^{n}}-\sigma^{*}\right| \leq \frac{C}{l} .
$$

Moreover we have

$$
\sigma^{*}=\lim _{L \rightarrow \infty}\left[\min _{(u, \mathbf{b}) \in \mathcal{A}_{0}\left(Q_{L}\right)} \frac{E\left(u, \mathbf{b}, Q_{L}\right)}{L^{n}}\right]
$$


In view of (1.10), we may interpret $\sigma^{*}$ as the ground state energy density. In fact, as a byproduct of the proof of Theorem 1.1, we will also show that the same limit holds true in (1.10) if we replace the Neumann condition in the definition of $\mathcal{A}_{0}$ with free boundary conditions. This amounts to defining $G$ in (1.2) to be the Green's function for $-\triangle$ with Dirichlet boundary conditions.

Let us remark that the decay rate in (1.9) is optimal in the sense that for a periodic pair $\left(u_{\text {per }}, \mathbf{b}_{\text {per }}\right)$ we have

$$
\limsup _{l \rightarrow \infty} l\left|\frac{E\left(u_{\text {per }}, \mathbf{b}_{\text {per }}, Q_{l}(a)\right)}{l^{n}}-\sigma\left(u_{\text {per }}, \mathbf{b}_{\text {per }}\right)\right|>0,
$$

where $\sigma\left(u_{\text {per }}, \mathbf{b}_{\text {per }}\right)$ denotes the energy per unit period.

We also prove that minimizers exhibit an equipartition of the surface and interaction energies with the uniform distribution estimate holding separately for each, though with a worse rate of decay.

Theorem 1.2. There exist positive constants $C$ and $c$, depending only on the volume fraction $m$ and space dimension $n$, with the following property: If $\left(u_{0}, \mathbf{b}_{0}\right)$ is a minimizer of $E\left(u, \mathbf{b}, Q_{L}\right)$ on $\mathcal{A}_{0}\left(Q_{L}\right)$, then for every $l \geq c$ and $a \in Q_{L}$ such that $Q_{l}(a) \subset Q_{L}$, we have

$$
\left.\left|\frac{1}{l^{n}} \int_{Q_{l}(a)} \frac{1}{2}\right| \mathbf{b}_{0}\right|^{2} d x-\frac{\sigma^{*}}{3}|+| \frac{1}{l^{n}} \int_{Q_{l}(a)}\left|\nabla u_{0}\right|-\frac{2 \sigma^{*}}{3} \mid \leq \frac{C}{\sqrt{l}} .
$$

Theorems 1.1 and 1.2 show that the energy of the optimal structure is uniformly distributed on a set scale. This inherent length scale is implicitly based on the constant $\sigma^{*}$, and, in order to set aside boundary and physical domain size effects, is exactly captured in the limit of $L \rightarrow \infty$.

The optimality of the decay rate in (1.9) might seem to suggest that minimizers should be nearly periodic. However, one could have structures which share these energy estimates but are hardly periodic ( $c f$. the so-called random checkerboard pattern discussed in Remark 6.2). Let us pursue this further by considering another approach, that of large scale density variations of the pattern. To this end, let $\phi: \mathbb{R}^{n} \rightarrow \mathbb{R}$ be a standard mollification kernel, i.e., a smooth function such that

$$
\phi \geq 0, \quad \phi=0 \text { outside } B_{1}(0), \quad \int_{\mathbb{R}^{n}} \phi d x=1 .
$$

For $R>0$ and $a \in \mathbb{R}^{n}$, let

$$
\phi_{R}(x):=\frac{1}{R^{n}} \phi\left(\frac{x-a}{R}\right) .
$$

Consider any admissible $(u, \mathbf{b})$ on $\mathcal{A}_{0}\left(Q_{L}\right)$ for a very large value of $L$. For $B_{R}(a) \subset$ $Q_{L}$, consider

$$
\int_{B_{R}(a)} \phi_{R}(x)(u(x)-m) d x .
$$

This is a measure of average volume-fraction fluctuations (or density variations) in a subsystem of size $R$ where $1 \ll R \ll L$. If the pattern is exactly periodic, then one finds that such fluctuations decay exponentially fast. More precisely, if $\left(u_{\text {per }}, \mathbf{b}_{\text {per }}\right)$ are periodic, then for $R$ sufficiently large, we have (cf. Proposition 6.1)(i))

$$
\int_{B_{R}(a)} \phi_{R}(x)\left(u_{\mathrm{per}}(x)-m\right) d x=O\left(\frac{1}{R^{p}}\right) \quad \text { for every } p>1 \text {. }
$$


On the other hand, let $\left(u_{0}, \mathbf{b}_{0}\right)$ be a minimizer of $E$ on $Q_{L}$ with $\mathbf{b}_{0}=-\nabla v$ for some $v \in H^{1}\left(Q_{L}\right)$. The basis of proving Theorem 1.1 will be a uniform $L^{\infty}$ bound (Lemma 3.6) on $\mathbf{b}_{0}$. Thus

$$
\begin{aligned}
\left|\int_{B_{R}(a)} \phi_{R}(x)\left(u_{0}(x)-m\right) d x\right| & =\left|\int_{B_{R}(a)} \phi_{R}(x) \Delta v d x\right| \\
& =\left|\int_{B_{R}(a)} \nabla \phi_{R}(x) \cdot \nabla v d x\right| \\
& \leq C \int_{B_{R}(a)}\left|\nabla \phi_{R}(x)\right| d x \\
& \leq \frac{C}{R} \int_{B_{1}(a)}|\nabla \phi(x)| d x \\
& \leq \frac{C}{R} .
\end{aligned}
$$

The contrast between (1.14) and (1.15) highlights that there is a gap between the behavior of a periodic pattern and what we are able to prove with Theorem 1.1. However, based upon Theorem 1.1 we will establish Theorem 1.3 below, which proves that not only $\mathbf{b}_{0}$ but also the associated potential $v$, where $\mathbf{b}_{0}=-\nabla v$, are uniformly bounded. This allows us to integrate by parts once more in (1.15) to obtain a better decay rate of $1 / R^{2}$, one step closer to periodicity ( $c f$. Proposition 6.1(ii)).

Theorem 1.3. There exist positive constants $C$ and $c$, depending only on the volume fraction $m$ and space dimension $n$, with the following property: If $\left(u_{0}, \mathbf{b}_{0}\right)$ is a minimizer of $E\left(u, \mathbf{b}, Q_{L}\right)$ on $\mathcal{A}_{0}\left(Q_{L}\right)$ with $L \geq c$, then there exists $v \in H^{1}\left(Q_{L}\right)$ such that $\mathbf{b}_{0}=-\nabla v$, and for all $x \in Q_{L}$,

$$
|v(x)| \leq C .
$$

As we will explain in Section 6, this better decay rate allows us (in dimension $n<4$ ), to reinforce the notion of uniform structure for minimizers by ruling out certain structures whose energy would also be uniformly distributed on a set scale, but whose pattern within a set cell is random, for example a random checkerboard pattern ( $c f$. Proposition 6.1(iii)).

As we have already pointed out, problem (1.7) is perhaps the simplest nonlocal problem to address short and long-range competitions. Surprisingly there has been little rigorous work addressing the structure of minimizers in $\mathbb{R}^{n}$, with $n>1$. Recently, however, this functional (and its diffuse interface counterpart) has appeared increasingly in the mathematical literature because of the relevance of its diffuse interface version to a model for microphase separation of diblock copolymers. In particular, there has been work in one space dimension (see for example [19, 20, 22, 4]), on rigorous scaling laws ([6]), and also much work, especially by Ren and Wei, on local minimizers (see for example [23, 24, 5, 10]).

The paper is organized as follows. In Section 2, we introduce the relevant notation and definitions. Section 3 begins with a brief sketch of the proof of Theorem 1.1 and how it is broken down into a (mostly) progressive series of lemmas. We then state precisely these lemmas, for which Theorem 1.1 is a direct consequence, and provide a proof of Theorem 1.2. The proofs of the lemmas comprise Section 4 
wherein the arguments for the crucial Lemmas 3.4 and 3.5 are reminiscent of Theorem 2.1 in [11], where an anisotropic $(1+1)$-problem is treated. Finally the proof and consequences of Theorem 1.3 are reserved for Sections 5 and 6 respectively.

\section{BAsic NOTATION AND DEFinitions}

We begin with an important comment on constants. Throughout this paper, when we use the word constant, we mean a constant which may depend on the volume fraction $m$ and the space dimension $n$, but on these parameters only. In the sequel, we suppress this dependence.

- We will use indexed notation such as $C_{0}, C_{1}$, etc. to denote constants that we wish to keep track of within a particular section and, for example, reference in different lemmas.

- We will use $C$ to explicitly denote a constant whose value, depending on the context, may change within a particular proof. When arguing with a particular sufficiently large $C$, we occasionally use $1 / C$ to denote a sufficiently small constant.

- When there is no reason to explicitly denote the constant, we adopt the notation $\lesssim$ and $\gtrsim$ for inequalities up to a constant. In principle, we adopt this notation whenever possible. However, it is sometimes more convenient for expository purposes to explicitly use the $C$.

Unless stated otherwise, $x=\left(x_{1}, \ldots, x_{n}\right)$ denotes a point in $\mathbb{R}^{n}$. By $d x$ we mean $n$-dimensional Lebesgue measure, and when it is clear we are integrating with respect to Lebesgue measure, we often leave the $d x$ out. For $\Omega \subset \mathbb{R}^{n}$ and $u \in B V(\Omega), \int_{\Omega}|\nabla u|$ denotes the total variation measure evaluated at $\Omega$. By $B_{r}(a)$ we mean the $n$-dimensional ball with centre $a \in \mathbb{R}^{n}$ and radius $r>0$.

For $Q_{L}$ defined by (1.8), $\nu$ denotes the normal to $\partial Q_{L}$ which always points in the positive axis direction; that is, on each face of $Q_{L}$ of the form $x_{i}= \pm L / 2, \nu_{i}=1$ and $\nu_{j}=0$ for $j \neq i$. We use $\overline{Q_{L}}$ to denote the closure of $Q_{L}$. For $u \in B V\left(Q_{L}, \pm 1\right)$ and $\mathbf{b} \in L^{2}\left(Q_{L}, \mathbb{R}^{n}\right)$, the differential equation $\operatorname{div} \mathbf{b}=u-m$ is always taken in the sense of distributions. When we write $\operatorname{div} \mathbf{b}+m \in[-1,1]$, we mean $\operatorname{div} \mathbf{b}+m \in L^{\infty}$ taking values in $[-1,1]$ a.e. For any pair $(\mathbf{b}, u)$ with $\operatorname{div} \mathbf{b}=u-m$, we note that $\mathbf{b} \cdot \nu$ has a well-defined trace on the boundary of any cube contained in $Q_{L}$. In general the trace is not a well-defined function but an element of the negative Sobolev space $H^{-1 / 2}$, and throughout this article $\mathbf{b} \cdot \nu$ on the boundary of any cube is interpreted in this trace sense. However, for our purposes, the details of the space $H^{-1 / 2}$ are not important, as we shall use trace estimates only for minimizers of $E$ : Hence $\mathbf{b}=-\nabla v$, where $v$ solves a Poisson equation, and the trace of $\mathbf{b} \cdot \nu$ will in fact be in $L^{2}$ (cf. Remark 2.1).

We will often use mollification. To fix notation, let $\phi$ be the standard mollification kernel of (1.11). For $r>0$, let $\phi_{r}$ be defined by (1.12) with $r$ in place of $R$ and $a=0$. For any function $f$ we define its mollification with $\phi_{r}$ by

$$
f_{r}^{*}(x):=\left(f * \phi_{r}\right)(x)=\int f(x-y) \phi_{r}(y) d y,
$$

with the understanding that the domain of $f$ must be sufficiently large so that the above integral makes sense. 
We need the following definitions of optimal densities. We define (cf. Remark[2.1)

$$
\begin{aligned}
\sigma\left(Q_{L}\right) & :=\min _{(u, \mathbf{b}) \in \mathcal{A}} \frac{1}{L^{n}} E\left(u, \mathbf{b}, Q_{L}\right), \\
\sigma^{0}\left(Q_{L}\right) & :=\min _{(u, \mathbf{b}) \in \mathcal{A}_{0}} \frac{1}{L^{n}} E\left(u, \mathbf{b}, Q_{L}\right),
\end{aligned}
$$

where

$$
\begin{gathered}
E\left(u, \mathbf{b}, Q_{L}\right):=\int_{Q_{L}}|\nabla u|+\frac{1}{2} \int_{Q_{L}}|\mathbf{b}|^{2} d x \\
\mathcal{A}\left(Q_{L}\right):=\left\{(u, \mathbf{b}) \mid u \in B V\left(Q_{L}, \pm 1\right), \mathbf{b} \in L^{2}\left(Q_{L}, \mathbb{R}^{n}\right), \operatorname{div} \mathbf{b}=u-m\right\} \\
\mathcal{A}_{0}\left(Q_{L}\right):=\left\{(u, \mathbf{b}) \mid u \in B V\left(Q_{L}, \pm 1\right), \mathbf{b} \in L^{2}\left(Q_{L}, \mathbb{R}^{n}\right)\right. \\
\left.\operatorname{div} \mathbf{b}=u-m, \mathbf{b} \cdot \nu=0 \text { on } \partial Q_{L}\right\} .
\end{gathered}
$$

It will also be necessary to consider optimal densities for $E$ with prescribed nonzero boundary conditions on $\mathbf{b} \cdot \nu$. In fact, it will be convenient to have such a density on rectangular boxes as well. To this end, we denote by $R_{L}$ any rectangular box of size $L$ which is not too anisotropic; that is, any set of the form

$$
R_{L}:=\left(a_{1}, b_{1}\right) \times \cdots \times\left(a_{n}, b_{n}\right),
$$

where $a_{i}, b_{i}$ satisfy

$$
\frac{1}{2} L \leq b_{1}-a_{1}, \ldots, b_{n}-a_{n} \leq L
$$

and we denote by $\left|R_{L}\right|$ its $n$-dimensional volume. For $g \in L^{2}\left(\partial R_{L}\right)$, we define

$$
\sigma^{g}\left(R_{L}\right):=\min _{(u, \mathbf{b}) \in \mathcal{A}_{g}} \frac{1}{\left|R_{L}\right|} E\left(u, \mathbf{b}, R_{L}\right),
$$

where

$$
\begin{aligned}
\mathcal{A}_{g}\left(R_{L}\right):=\left\{(u, \mathbf{b}) \mid u \in B V\left(R_{L}, \pm 1\right), \mathbf{b} \in L^{2}\left(R_{L}, \mathbb{R}^{n}\right),\right. & \\
& \left.\operatorname{div} \mathbf{b}=u-m, \mathbf{b} \cdot \nu=g \text { on } \partial R_{L}\right\} .
\end{aligned}
$$

Remark 2.1. Note that the direct method of the calculus of variations implies that the densities $\sigma^{0}\left(Q_{L}\right)$ and $\sigma\left(Q_{L}\right)$ are attained by at least one optimal pair $\left(u_{0}, \mathbf{b}_{0}\right)$. By properties of the trace space $H^{-1 / 2}$, this is also the case for $\sigma^{g}\left(R_{L}\right)$ provided $\mathcal{A}_{g}\left(R_{L}\right) \neq \emptyset$, i.e., provided the boundary data $g$ is sufficiently small to be compatible with the fact that, for some $u \in\{-1,1\}$,

$$
\int_{\partial R_{L}} g=\int_{R_{L}} u-m \leq 2 L^{n} .
$$

Note that when using $\sigma^{g}$ (cf. Lemmas 3.4 and 3.7), we will always impose a suitable smallness condition on $g$ consistent with (2.3).

Moreover, following the basic arguments of (1.4), one sees that the optimal $\mathbf{b}_{0}$ for either $\sigma^{0}$ or $\sigma^{g}$ is a gradient: That is, $\mathbf{b}_{0}=-\nabla v$ for $v$ solving $-\triangle v=u_{0}-m$ with $\mathbf{b}_{0} \cdot \nu=0$ or $\mathbf{b}_{0} \cdot \nu=g$ respectively on the boundary; in the case of free 
boundary conditions (i.e., for $\sigma$ ), the same holds true with the natural boundary conditions being Dirichlet conditions for $v$ (i.e., $v=0$ on the boundary).

A key ingredient in the proof of Theorem 1.1, which will circumvent the need for optimal constructions, is the relaxed problem associated with $\sigma^{g}$ :

$$
\begin{aligned}
\inf \left\{\int_{R_{L}} \frac{1}{2}|\mathbf{b}|^{2} d x \mid\right. & \mathbf{b} \in L^{2}\left(R_{L}, \mathbb{R}^{n}\right), \\
& \left.\operatorname{div} \mathbf{b}+m \in[-1,1] \text { in } R_{L}, \mathbf{b} \cdot \nu=g \text { on } \partial R_{L}\right\} .
\end{aligned}
$$

Note that we may again invoke the direct method in the calculus of variations to show that a minimizer for the relaxed problem does indeed exist; hence the infimum above could be replaced with a minimum.

\section{The Proofs of Theorems 1.1 And 1.2}

Theorem 1.1 is a direct consequence of two lemmas which are, in turn, consequences of other lemmas. Theorem 1.2 will directly follow from Theorem 1.1 by a simple rescaling argument. Thus let us first focus on outlining the proof of Theorem 1.1, and how it is broken down into a progressive series of lemmas involving the densities $\sigma, \sigma^{0}$ and $\sigma^{g}$. The reader is encouraged to read this sketch in consultation with the statements of the lemmas which follow.

Sketch of the proof of Theorem 1.1. In Lemma 3.1, we start with some elementary inequalities involving the two minimal energies per volume, namely $\sigma$ for the free boundary condition and $\sigma^{0}$ for the zero-flux boundary condition. Trivially, the free boundary condition admits a lower energy than the zero-flux boundary condition (Lemma 3.1(i)). By cutting and pasting, one easily sees that $\sigma\left(Q_{l}\right)$ is essentially increasing in the size $l \gg 1$ of the cube, while $\sigma^{0}\left(Q_{l}\right)$ is essentially decreasing (Lemma 3.1(ii) and (iii)). A straightforward construction and an elementary interpolation argument show that $\sigma^{0}\left(Q_{l}\right)$ is bounded above and $\sigma\left(Q_{l}\right)$ is bounded below uniformly in $l \gg 1$ (Lemma 3.1(iv) and (v)).

With Lemma 3.1 in hand, we will be able to establish the existence of the limit of (1.10), or more precisely Lemma 3.10, by elementary calculus arguments if we can bound $\sigma^{0}$ by $\sigma$ on cubes of comparable size. The appropriate bound is stated in Lemma 3.8. Together with Lemma 3.9, Lemma 3.8 also yields (1.9). Hence Lemmas 3.8 and 3.9 are the core technical statements leading up to Theorem 1.1 For the remainder of this discussion we focus on Lemma 3.8 and its derivation from Lemmas 3.2 - 3.7.

The heart of Lemma 3.8 rests on, given a minimizer $\left(u_{0}, \mathbf{b}_{0}\right)$ of $\sigma$, constructing an appropriate comparison function $(u, \mathbf{b})$ for $\sigma^{0}$. This means that we need to construct a $\mathbf{b}$ with zero-flux boundary conditions from a $\mathbf{b}_{0}$ with generically nonvanishing flux $g=\mathbf{b}_{0} \cdot \nu$ at the boundary at hand. Rephrasing the problem, we need to construct, on cubes of comparable size, an appropriate comparison function $(u, \mathbf{b})$ for $\sigma^{0}$ given a minimizer $\left(u_{0}, \mathbf{b}_{0}\right)$ of $\sigma^{g}$. This is done in Lemma 3.7 by a construction in a boundary layer, which relies on an $L^{\infty}$-bound on $g$. In fact, $\Lambda:=\|g\|_{L^{\infty}}$ is the width of the boundary layer and thus determines the quality of the estimate.

Thus we need to know that for a minimizer $\left(u_{0}, \mathbf{b}_{0}\right)$ of $\sigma\left(Q_{l}\right)$, the flux $g=\mathbf{b}_{0} \cdot \nu$ at the boundary satisfies an $L^{\infty}$-bound uniform in $l$. This is the purpose of Lemma 3.6, where we show that $\mathbf{b}_{0}$ itself satisfies an $L^{\infty}$-bound uniform in $l$. The $L^{\infty}$-bound 
on $\mathbf{b}_{0}$ follows from a bound on $l^{-n} \int_{Q_{l}(a) \cap Q_{L}}\left|\mathbf{b}_{0}\right|^{2}$ that is uniform in $l \gg 1$ and $a$. The argument relies on elementary elliptic regularity theory applied to $\mathbf{b}_{0}=-\nabla v_{0}$ which satisfies $-\triangle v_{0}=u_{0}-m \in[-2,2]$. The bound on $l^{-n} \int_{Q_{l}(a) \cap Q_{L}}\left|\mathbf{b}_{0}\right|^{2}$ is contained in the bound on the energy density $l^{-n} E\left(u_{0}, \mathbf{b}_{0}, Q_{l}(a) \cap Q_{L}\right)$ stated in Lemma 3.5, which is a weakened version of Theorem 1.1. Here we show that the energy density is uniformly bounded - albeit not by the asymptotically optimal constant $\sigma^{*}$.

Roughly speaking, the merit of Lemma 3.5 is to convert the global energy bound $E\left(u_{0}, \mathbf{b}_{0}, Q_{L}\right) \leq C L^{n}$ of the minimizer $\left(u_{0}, \mathbf{b}_{0}\right)$ (with free boundary conditions, say) into the local energy bound $E\left(u_{0}, \mathbf{b}_{0}, Q_{l}(a) \cap Q_{L}\right) \leq C l^{n}$ on subcubes $Q_{l}(a)$ with $a \in Q_{L}$. This is done by an ode argument in $l \mapsto E\left(u_{0}, \mathbf{b}_{0}, Q_{l}(a) \cap Q_{L}\right)$. To derive the appropriate nonlinear differential inequality, we glue in the optimal construction for $\sigma^{g}\left(Q_{l}(a) \cap Q_{L}\right)$ with $g$ taken to be $\mathbf{b}_{0} \cdot \nu$ on $\partial Q_{l}(a) \cap Q_{L}$. This yields a competitor $(u, \mathbf{b})$ for $\left(u_{0}, \mathbf{b}_{0}\right)$, and thus allows us to estimate $E\left(u_{0}, \mathbf{b}_{0}, Q_{l}(a) \cap Q_{L}\right)$ in terms of $\sigma^{g}\left(Q_{l}(a) \cap Q_{L}\right)$ (and a perimeter term of lower order). We then use Lemma 3.4 to estimate $\sigma^{g}\left(Q_{l}(a) \cap Q_{L}\right)$ in terms of a nonlinear expression in $\int_{\partial Q_{l}(a) \cap Q_{L}}\left|\mathbf{b}_{0} \cdot \nu\right|^{2}$ and notice that $\int_{\partial Q_{l}(a) \cap Q_{L}}\left|\mathbf{b}_{0} \cdot \nu\right|^{2}$ itself is bounded by $\frac{d}{d l} E\left(u_{0}, \mathbf{b}_{0}, Q_{l}(a) \cap Q_{L}\right)$. This approach is reminiscent of [1] and yields the desired nonlinear differential inequality.

Hence the crucial ingredient for Lemma 3.5 is Lemma 3.4 where it is shown that on some not too anisotropic rectangle $R_{L}\left(R_{L}=Q_{l}(a) \cap Q_{L}\right.$ in the application to Lemma 3.5), the minimal energy per volume $\sigma^{g}$ for inhomogeneous flux data $g$ on $\partial R_{L}$ is estimated in terms of the $L^{2}$-norm of $g$. It is crucial for Lemma 3.5 that the exponent $\frac{n+2}{n+1}$ is strictly less than the isoperimetric exponent $\frac{n}{n-1}$.

In order to derive Lemma 3.4, we first establish the corresponding bound for the relaxed problem (2.4) in Lemma 3.3(b). Lemma 3.4 follows easily from Lemma 3.3 (b): We take the optimal $\mathbf{b}_{0}$ from the relaxed problem and replace the function $\operatorname{div} \mathbf{b}_{0}+m$, which takes values in $[-1,1]$, by a function $u$ with values \pm 1 that is piecewise constant on rectangles of diameter of order 1 and is of the same local volume fraction. The surface energy and the additional bulk energy are of the order $L^{n}$.

The relaxed variational problem (2.4) is convex and thus admits a dual formulation on the level of the potential $v$, cf. Lemma 3.3(a). The dual formulation allows us to reduce the upper bound on the energy of the relaxed problem to a trace estimate, stated in Lemma 3.2. It is the scaling of this trace estimate in the boundary layer thickness $\epsilon$ which sets the crucial exponent in Lemma 3.3.(b) and thus Lemma 3.4 .

We now present the precise statements of the lemmas involved in the proof of Theorems 1.1. Proofs are postponed to the next section.

Lemma 3.1 (Basic inequalities). There exists a constant $C<\infty$ such that for $l \geq C$,

(i) $\sigma\left(Q_{l}\right) \leq \sigma^{0}\left(Q_{l}\right)$;

(ii) $\forall k \in \mathbb{N}, \sigma\left(Q_{k l}\right) \geq \sigma\left(Q_{l}\right)$;

(iii) $\forall k \in \mathbb{N}, \quad \sigma^{0}\left(Q_{k l}\right) \leq \sigma^{0}\left(Q_{l}\right)+\frac{C}{l}$;

(iv) $\sigma^{0}\left(Q_{l}\right) \leq C$;

(v) $\sigma\left(Q_{l}\right) \geq \frac{1}{C}$. 
Lemma 3.2 (Trace estimate). For any $v \in H^{1}\left((0,1)^{n}\right)$ and $0<\epsilon \leq 1$, we have

$$
\int_{\{0\} \times(0,1)^{n-1}}|v|^{2} d x^{\prime} \lesssim \epsilon \int_{(0, \epsilon) \times(0,1)^{n-1}}|\nabla v|^{2} d x+\left(\frac{1}{\epsilon^{(n+1) / 2}} \int_{(0, \epsilon) \times(0,1)^{n-1}}|v| d x\right)^{2} .
$$

Lemma 3.3 (Duality and upper bound for the relaxed problem).

(a) We have the following duality relation for the relaxed problem (2.4): For every rectangular box $R_{L}$ and $g \in L^{2}\left(\partial R_{L}\right)$ there holds

$$
\begin{aligned}
& \inf \left\{\int_{R_{L}} \frac{1}{2}|\mathbf{b}|^{2} \mid(\operatorname{div} \mathbf{b}+m) \in[-1,1] \text { in } R_{L}, \mathbf{b} \cdot \nu=g \text { on } \partial R_{L}\right\} \\
& \quad=-\inf \left\{\int_{R_{L}} \frac{1}{2}|\nabla v|^{2}+\int_{R_{L}}(m v+|v|)+\int_{\partial R_{L}} g v \mid v \in H^{1}\left(R_{L}\right)\right\} .
\end{aligned}
$$

(b) Let $\mathbf{b} \in L^{2}\left(R_{L}, \mathbb{R}^{n}\right)$ be a minimizer of the relaxed problem (2.4). There exists a constant $C_{0}$ with the following property: For every rectangular box $R_{L}$ satisfying (2.2) and every $g \in L^{2}\left(\partial R_{L}\right)$ such that

$$
\int_{\partial R_{L}} g^{2} \leq \frac{1}{C_{0}} L^{n+1}
$$

we have

$$
\int_{R_{L}}|\mathbf{b}|^{2} \leq C_{0}\left(\int_{\partial R_{L}} g^{2}\right)^{\frac{n+2}{n+1}} .
$$

Lemma 3.4 (Upper bound for $\sigma^{g}\left(R_{L}\right)$ ). There exists a constant $C_{1}$ with the following property: For every rectangular box $R_{L}$ satisfying (2.2) with $L \geq 1$, and $g \in L^{2}\left(\partial R_{L}\right)$ such that

$$
\int_{\partial R_{L}} g^{2} \leq \frac{1}{C_{1}} L^{n+1}
$$

we have

$$
\sigma^{g}\left(R_{L}\right) \leq C_{1}\left(1+\frac{1}{L^{n}}\left(\int_{\partial R_{L}} g^{2}\right)^{\frac{n+2}{n+1}}\right) .
$$

Lemma 3.5 (Uniform energy distribution - first version). There exists a constant $C_{2}$ with the following property: Let $\left(u_{0}, \mathbf{b}_{0}\right)$ be a minimizer of $E\left(u, \mathbf{b}, Q_{L}\right)$ on either $\mathcal{A}\left(Q_{L}\right)$ or $\mathcal{A}_{0}\left(Q_{L}\right)$. Then for all centres $a \in Q_{L}$ and $L \geq l \geq C_{2}$,

$$
E\left(u_{0}, \mathbf{b}_{0}, Q_{l}(a) \cap Q_{L}\right) \leq 3 C_{1} l^{n},
$$

with $C_{1}$ as in Lemma 3.4 .

Lemma 3.6 ( $L^{\infty}$-bound for minimizers). There exists a constant $C$ with the following property: Let $\left(u_{0}, \mathbf{b}_{0}\right)$ be a minimizer of $E\left(u, \mathbf{b}, Q_{L}\right)$ on $\mathcal{A}\left(Q_{L}\right)$ or $\mathcal{A}_{0}\left(Q_{L}\right)$; then we have

$$
\left|\mathbf{b}_{0}\right| \leq C
$$

Lemma 3.7 (Comparison of $\sigma^{0}$ and $\sigma^{g}$ ). There exist constants $C_{3}, C$ with the following property: For every $g: \partial Q_{L} \rightarrow \mathbb{R}$ with

$$
\Lambda:=\sup _{\partial Q_{L}}|g| \leq \frac{1}{2 C_{3}} L
$$


we have

$$
\begin{aligned}
& \text { (i) } \sigma^{g}\left(Q_{L}\right) \leq \sigma^{0}\left(Q_{L-2 C_{3} \Lambda}\right)+\frac{C\left(1+\Lambda^{3}\right)}{L}, \\
& \text { (ii) } \sigma^{0}\left(Q_{L+2 C_{3} \Lambda}\right) \leq \sigma^{g}\left(Q_{L}\right)+\frac{C\left(1+\Lambda^{3}\right)}{L} .
\end{aligned}
$$

Lemma 3.8 (Comparison of $\sigma^{0}$ and $\sigma$ ). There exist constants $C_{4}, C$ such that

$$
\sigma^{0}\left(Q_{L+C_{4}}\right) \leq \sigma\left(Q_{L}\right)+\frac{C}{L}
$$

for all $L \geq C_{4}$.

Lemma 3.9 (Uniform energy distribution - second version). There exist constants $C_{5}, C$ with the following property: Let $\left(u_{0}, \mathbf{b}_{0}\right)$ be a minimizer of $E\left(u, \mathbf{b}, Q_{L}\right)$ on $\mathcal{A}_{0}\left(Q_{L}\right)$. Then for any $Q_{l}(a) \subset Q_{L}$ with $l \geq C_{5}$, we have

$$
\frac{E\left(u_{0}, \mathbf{b}_{0}, Q_{l}\right)}{l^{n}} \leq \sigma^{0}\left(Q_{l-C_{5}}\right)+\frac{C}{l}
$$

Lemma 3.10 (Large $l$ behavior of $\sigma(l), \sigma^{0}(l)$ ). There exist two constants $\sigma^{*} \in$ $(0, \infty)$ and $C<\infty$ such that for all $l \geq C$,

$$
\sigma^{*}-\frac{C}{l} \leq \sigma\left(Q_{l}\right) \leq \sigma^{0}\left(Q_{l}\right) \leq \sigma^{*}+\frac{C}{l} .
$$

Proof of Theorem 1.1. Theorem 1.1] is now a direct consequence of Lemmas 3.9] and 3.10 .

Proof of Theorem 1.2. Let $\left(u_{0}, \mathbf{b}_{0}\right)$ be a minimizer of $E\left(u, \mathbf{b}, Q_{L}\right)$ on $\mathcal{A}_{0}\left(Q_{L}\right)$, and let $Q_{l}(a) \subset Q_{L}$. By Theorem 1.1, there exists constants $C$ and $c$ such that

$$
\left|\frac{1}{l^{n}} E\left(u_{0}, \mathbf{b}_{0}, Q_{l}(a)\right)-\sigma^{*}\right| \leq \frac{C}{l}
$$

when $l \geq c$. For every $\lambda \in[1 / 2,3 / 2]$ we consider the rescaled couple $\left(u_{0}^{\lambda}, \mathbf{b}_{0}^{\lambda}\right)$ defined by

$$
u_{0}^{\lambda}(\lambda x):=u_{0}(x) \quad \text { and } \quad \frac{1}{\lambda} \mathbf{b}_{0}^{\lambda}(\lambda x):=\mathbf{b}_{0}(x) .
$$

One easily checks that $\left(u_{0}^{\lambda}, \mathbf{b}_{0}^{\lambda}\right)$ belongs to $\mathcal{A}_{0}\left(Q_{\lambda L}\right)$, and therefore Theorem 1.1 also implies

$$
\frac{1}{\lambda^{n} l^{n}} E\left(u_{0}, \mathbf{b}_{0}, Q_{\lambda l}(\lambda a)\right)-\sigma^{*} \geq-\frac{C}{\lambda l} \geq-\frac{2 C}{l}
$$

for $\lambda l \geq c$. For the rest of the proof we assume that $l \geq 2 c$; hence (3.5) and (3.6) hold for every $\lambda$.

Next we define

$$
f(\lambda):=\frac{1}{\lambda^{n} l^{n}} E\left(u_{0}, \mathbf{b}_{0}, Q_{\lambda l}(\lambda a)\right) .
$$

Then (3.5) and (3.6) imply that

$$
f(\lambda) \geq \sigma^{*}-\frac{2 C}{l} \geq f(1)-\frac{3 C}{l} .
$$

Moreover a simple computation yields

$$
f(\lambda)=a \lambda^{2}+b \lambda^{-1},
$$


where

$$
a:=\frac{1}{l^{n}} \int_{Q_{l}(a)} \frac{1}{2}\left|\mathbf{b}_{0}\right|^{2} d x \quad \text { and } \quad b:=\frac{1}{l^{n}} \int_{Q_{l}(a)}\left|\nabla u_{0}\right| .
$$

Note that $a+b=f(1)=l^{-n} E\left(u_{0}, \mathbf{b}_{0}, Q_{l}(a)\right)$, and therefore (3.5) can be rewritten as

$$
\left|a+b-\sigma^{*}\right| \leq \frac{C}{l}
$$

Now we apply the following elementary statement (we omit the proof): Let $f:[1 / 2,3 / 2] \rightarrow \mathbb{R}$ be a function of class $C^{2}$ and let $\delta, m$ be positive constants such that

$$
f(\lambda) \geq f(1)-\delta \quad \text { and } \quad f^{\prime \prime}(\lambda) \leq 2 m
$$

for every $\lambda \in[1 / 2,3 / 2]$. If $\delta \leq m / 4$, then

$$
\left|f^{\prime}(1)\right| \leq 2 \sqrt{m \delta} .
$$

Let us check the assumptions: the first inequality in (3.12) follows from (3.8) provided we set $\delta:=3 C / l$; the second inequality in (3.12) follows from the estimate

$$
f^{\prime \prime}(\lambda)=2 a+2 b \lambda^{-3} \leq 16(a+b) \leq 16\left(\sigma^{*}+C\right)=: 2 m,
$$

where the last inequality follows from (3.11) provided that $l \geq 1$; finally, assumption $\delta \leq m / 4$ is verified for $l \geq 12 C / m$. Note that the assumptions on $l$ so far add up to $l \geq \max \{2 c, 1,12 C / m\}$.

Taking into account (3.9), conclusion (3.13) becomes

$$
|2 a-b| \lesssim \frac{1}{\sqrt{l}}
$$

and recalling (3.11) we get

$$
\left|3 a-\sigma^{*}\right| \leq\left|a+b-\sigma^{*}\right|+|2 a-b| \lesssim \frac{1}{\sqrt{l}} .
$$

In a similar way one obtains $\left|2 b-\sigma^{*}\right| \lesssim 1 / \sqrt{l}$, and the proof is complete.

\section{Proofs of the lemmas}

Proof of Lemma 3.1. Statement (i) is obvious by definition of $\sigma\left(Q_{l}\right)$ since $\mathcal{A}_{0}\left(Q_{l}\right) \subset$ $\mathcal{A}\left(Q_{l}\right)$.

For (ii), consider a minimizer $(u, \mathbf{b})$ for $\sigma\left(Q_{k l}\right)$ : For at least one of the $k^{n}$ subsquares of size $l$ of $Q_{k l}$, the energy of $(u, \mathbf{b})$ on this subsquare must be smaller than one $k^{n}$-th of the energy on the square $Q_{k l}$, and since the restriction of $(u, \mathbf{b})$ to this subsquare is admissible for $\sigma\left(Q_{l}\right)$, the inequality follows.

For (iii), let $(u, \mathbf{b})$ be a minimizer for $\sigma^{0}\left(Q_{l}\right)$, extend this pair by periodicity to $Q_{k l}$, and denote such an extension by $(\widehat{u}, \widehat{\mathbf{b}})$. Since $\mathbf{b} \cdot \nu=0$ on $\partial Q_{l}$, $(\widehat{u}, \widehat{\mathbf{b}})$ is admissible for $\sigma^{0}\left(Q_{k l}\right)$, and

$$
E\left(\widehat{u}, \widehat{\mathbf{b}}, Q_{k l}\right) \leq k^{n} E\left(u, b, Q_{l}\right)+C k^{n} l^{n-1},
$$

since we may introduce jumps in $\widehat{u}$ at the interfaces of the cubes of size $l$. Dividing both sides by $k^{n} l^{n}$, we have

$$
\sigma^{0}\left(Q_{k l}\right) \leq \frac{E\left(\widehat{u}, \widehat{\mathbf{b}}, Q_{k l}\right)}{k^{n} l^{n}} \leq \sigma^{0}\left(Q_{l}\right)+\frac{C}{l} .
$$


Statement (iv) follows by computating $E$ for a suitable test function $u$ and a compatible vector field $\mathbf{b}$; for example taking for $u$ a lamellar pattern with volume fraction $m$ and period 1 .

It is only the last statement (v) which is nontrivial. The proof involves an interpolation-type inequality. If we were concerned with either periodic or Neumann $(\mathbf{b} \cdot \nu=0)$ boundary conditions for $\mathbf{b}$, the result would directly follow from an interpolation inequality between $B V+H^{-1}$ and $L^{2}$; see for example Lemma 2.3 in [7. For the case of free boundary conditions, a slightly different inequality is needed. To this end, let $(u, \mathbf{b}) \in \mathcal{A}\left(Q_{l}\right)$, and define

$$
w:=u-m .
$$

Thus we have $\operatorname{div} \mathbf{b}=w$. Let $Q_{l}^{\prime \prime} \subset Q_{l}^{\prime}$ be $n$-dimensional cubes with the same centre as $Q_{l}$ but with side lengths $\frac{1}{2} l$ and $\frac{3}{4} l$ respectively. Let $\eta$ be a smooth $c u t$-off function such that

$$
\eta=1 \text { in } Q_{l}^{\prime \prime} \quad \text { and } \quad \eta=0 \text { in } Q_{l} \backslash Q_{l}^{\prime} .
$$

Without loss of generality, we may assume $l \geq 4$. On $Q_{l}^{\prime}$, consider the mollifications $w_{1}^{*}$ and $\mathbf{b}_{1}^{*}$ (i.e., (1.12) and (2.1) with $r=R=1$ and centre $a=0$ ). Note that, by assumption, we have $1<\operatorname{dist}\left(\partial Q_{l}, \partial Q_{l}^{\prime}\right)$. Since $\operatorname{div} \mathbf{b}=w$ on $Q$, we have $\operatorname{div} \mathbf{b}_{1}^{*}=w_{1}^{*}$ on $Q^{\prime}$. We show that the first and second terms of $E(u, \mathbf{b})$ are bounded below by $\int_{Q_{l}^{\prime}} \eta\left|w-w_{1}^{*}\right|^{2}$ and $\int_{Q_{l}^{\prime}} \eta\left|w_{1}^{*}\right|^{2}$ respectively.

Integrating by parts, we find

$$
\begin{aligned}
\int_{Q_{l}^{\prime}} \eta\left|w_{1}^{*}\right|^{2} & =\int_{Q_{l}^{\prime}} \eta\left(\operatorname{div} \mathbf{b}_{1}^{*}\right) w_{1}^{*} \\
& =-\int_{Q_{l}^{\prime}} \mathbf{b}_{1}^{*} \cdot \nabla\left(\eta w_{1}^{*}\right) \\
& =-\int_{Q_{l}^{\prime}} \mathbf{b} \cdot \nabla\left(\eta w_{1}^{*}\right)_{1}^{*} \\
& \leq\left(\int_{Q_{l}^{\prime}}|\mathbf{b}|^{2}\right)^{\frac{1}{2}}\left(\int_{Q_{l}^{\prime}}\left|\nabla\left(\eta w_{1}^{*}\right)_{1}^{*}\right|^{2}\right)^{\frac{1}{2}} \\
& \lesssim\left(\int_{Q_{l}^{\prime}}|\mathbf{b}|^{2}\right)^{\frac{1}{2}}\left(\int_{Q_{l}^{\prime}}\left(\eta w_{1}^{*}\right)^{2}\right)^{\frac{1}{2}},
\end{aligned}
$$

where in line (4.1) we used the Cauchy-Schwarz inequality, and in (4.2) we used the fact that $\|\nabla \phi\|_{\infty}$ depends only on the dimension $n$. Thus

$$
\int_{Q_{l}^{\prime}} \eta\left|w_{1}^{*}\right|^{2} \lesssim \int_{Q_{l}^{\prime}}|\mathbf{b}|^{2} .
$$

For the surface energy, we have

$$
\begin{aligned}
\int_{Q_{l}^{\prime}} \eta\left|w-w_{1}^{*}\right|^{2} & \lesssim \int_{Q_{l}^{\prime}} \eta\left|w-w_{1}^{*}\right| \\
& \lesssim \int_{Q_{l}^{\prime}}\left|w-w_{1}^{*}\right| \lesssim \int_{Q_{l}}|\nabla w|=\int_{Q_{l}}|\nabla u|
\end{aligned}
$$


Combining (4.3) and (4.4), we find

$$
\begin{aligned}
\int_{Q_{l}}|\nabla u|+\int_{Q_{l}}|\mathbf{b}|^{2} & \gtrsim \int_{Q_{l}^{\prime}} \eta\left|w_{1}^{*}\right|^{2}+\int_{Q_{l}^{\prime}} \eta\left|w-w_{1}^{*}\right|^{2} \\
& \geq \int_{Q_{l}^{\prime}} \eta|w|^{2} \gtrsim \int_{Q_{l}^{\prime}} \eta \gtrsim\left|Q_{l}^{\prime \prime}\right| \gtrsim l^{n} .
\end{aligned}
$$

Proof of Lemma 3.2, Let $v \in H^{1}\left((0,1)^{n}\right)$ and $0<\epsilon \leq 1$. The proof of (3.1) follows from two estimates. The first is the trace estimate

$$
\int_{\{0\} \times(0,1)^{n-1}}|v|^{2} d x^{\prime} \lesssim \epsilon \int_{(0, \epsilon) \times(0,1)^{n-1}}|\nabla v|^{2} d x+\int_{(0,1)^{n-1}}|\bar{v}|^{2} d x^{\prime},
$$

where $\bar{v}$ is defined by

$$
\bar{v}\left(x^{\prime}\right):=\frac{1}{\epsilon} \int_{0}^{\epsilon}\left|v\left(x_{1}, x^{\prime}\right)\right| d x_{1},
$$

where $x^{\prime}:=\left(x_{2}, \ldots, x_{n}\right) \in \mathbb{R}^{n-1}$. The second is the following $(n-1)$-dimensional Nash-type inequality (cf. [16]): For $w \in H^{1}\left((0,1)^{n-1}\right)$,

$$
\begin{aligned}
\int_{(0,1)^{n-1}}|w|^{2} d x \lesssim & \left(\int_{(0,1)^{n-1}}|\nabla w|^{2} d x\right)^{\frac{n-1}{n+1}}\left(\int_{(0,1)^{n-1}}|w| d x\right)^{\frac{4}{n+1}} \\
& +\left(\int_{(0,1)^{n-1}}|w| d x\right)^{2}
\end{aligned}
$$

We first prove (3.1) assuming (4.5) and (4.7). To this end, we apply (4.7) to $w\left(x^{\prime}\right)=\bar{v}\left(x^{\prime}\right)$. Note that by (4.6),

$$
\int_{(0,1)^{n-1}}|\bar{v}| d x^{\prime}=\frac{1}{\epsilon} \int_{(0, \epsilon) \times(0,1)^{n-1}}|v| d x \quad \text { and } \quad\left|\nabla \bar{v}\left(x^{\prime}\right)\right| \leq \frac{1}{\epsilon} \int_{0}^{\epsilon}\left|\nabla v\left(x_{1}, x^{\prime}\right)\right| d x_{1} .
$$

Hence by Jensen's inequality,

$$
\left|\nabla \bar{v}\left(x^{\prime}\right)\right|^{2} \leq \frac{1}{\epsilon} \int_{0}^{\epsilon}\left|\nabla v\left(x_{1}, x^{\prime}\right)\right|^{2} d x_{1}
$$

and so

$$
\int_{(0,1)^{n-1}}|\nabla \bar{v}|^{2} d x^{\prime} \leq \frac{1}{\epsilon} \int_{(0, \epsilon) \times(0,1)^{n-1}}|\nabla v(x)|^{2} d x .
$$

Thus (4.7) turns into

$$
\begin{aligned}
\int_{(0,1)^{n-1}}|\bar{v}|^{2} d x^{\prime} \lesssim & \left(\frac{1}{\epsilon} \int_{(0, \epsilon) \times(0,1)^{n-1}}|\nabla v|^{2} d x\right)^{\frac{n-1}{n+1}}\left(\frac{1}{\epsilon} \int_{(0, \epsilon) \times(0,1)^{n-1}}|v| d x\right)^{\frac{4}{n+1}} \\
& +\left(\frac{1}{\epsilon} \int_{(0, \epsilon) \times(0,1)^{n-1}}|v| d x\right)^{2} .
\end{aligned}
$$


Inserting this into (4.5), we find

$$
\begin{aligned}
\int_{\{0\} \times(0,1)^{n-1}}|v|^{2} d x^{\prime} & \lesssim \epsilon \int_{(0, \epsilon) \times(0,1)^{n-1}}|\nabla v|^{2} d x \\
& +\left(\frac{1}{\epsilon} \int_{(0, \epsilon) \times(0,1)^{n-1}}|\nabla v|^{2} d x\right)^{\frac{n-1}{n+1}}\left(\frac{1}{\epsilon} \int_{(0, \epsilon) \times(0,1)^{n-1}}|v| d x\right)^{\frac{4}{n+1}} \\
& +\left(\frac{1}{\epsilon} \int_{(0, \epsilon) \times(0,1)^{n-1}}|v| d x\right)^{2} .
\end{aligned}
$$

By Young's inequality, we have

$$
\begin{aligned}
& \left(\frac{1}{\epsilon} \int_{(0, \epsilon) \times(0,1)^{n-1}}|\nabla v|^{2} d x\right)^{\frac{n-1}{n+1}}\left(\frac{1}{\epsilon} \int_{(0, \epsilon) \times(0,1)^{n-1}}|v| d x\right)^{\frac{4}{n+1}} \\
& \quad=\left(\epsilon \int_{(0, \epsilon) \times(0,1)^{n-1}}|\nabla v|^{2} d x\right)^{\frac{n-1}{n+1}}\left(\epsilon^{-\frac{n+1}{2}} \int_{(0, \epsilon) \times(0,1)^{n-1}}|v| d x\right)^{2 \frac{2}{n+1}} \\
& \quad \lesssim \epsilon \int_{(0, \epsilon) \times(0,1)^{n-1}}|\nabla v|^{2} d x+\left(\epsilon^{-\frac{n+1}{2}} \int_{(0, \epsilon) \times(0,1)^{n-1}}|v| d x\right)^{2} .
\end{aligned}
$$

Inserting (4.9) into (4.8) implies the estimate (3.1) since $\epsilon \leq 1$.

It remains to prove (4.5) and (4.7). The estimate (4.5) is easy. It is based upon a standard one-dimensional trace estimate which yields

$$
\left|v\left(0, x^{\prime}\right)\right| \leq \int_{0}^{\epsilon}\left|\partial_{x_{1}} v\left(x_{1}, x^{\prime}\right)\right| d x_{1}+\frac{1}{\epsilon} \int_{0}^{\epsilon}\left|v\left(x_{1}, x^{\prime}\right)\right| d x_{1}
$$

for all $x^{\prime} \in(0,1)^{n-1}$. We square both sides of (4.10) and apply Jensen's inequality to find

$$
\left|v\left(0, x^{\prime}\right)\right|^{2} \leq 2 \epsilon \int_{0}^{\epsilon}\left|\partial_{x_{1}} v\left(x_{1}, x^{\prime}\right)\right|^{2} d x_{1}+2\left(\frac{1}{\epsilon} \int_{0}^{\epsilon}\left|v\left(x_{1}, x^{\prime}\right)\right| d x_{1}\right)^{2} .
$$

Integrating with respect to $x^{\prime}$ yields (4.5).

To prove the Nash-type estimate (4.7) we set $d=n-1$. Hence (4.7) reduces to

$$
\|w\|_{2} \lesssim\|w\|_{1}^{\frac{2}{d+2}}\|\nabla w\|_{2}^{\frac{d}{d+2}}+\|w\|_{1}
$$

where $\|w\|_{p}$ denotes the $L^{p}$ norm on $(0,1)^{d}$.

We begin with some preliminary estimates. For every $v \in W^{1,1}\left((0,1)^{d}\right)$, denote by $m(v)$ the average of $v$. It is well-known that $\|\nabla v\|_{1}+|m(v)|$ is equivalent to the usual $W^{1,1}$-norm, and therefore the Sobolev embedding theorem yields

$$
\|v\|_{1^{*}} \lesssim\|\nabla v\|_{1}+|m(v)|
$$

where $1^{*}=d /(d-1)$ is the Sobolev exponent (for $d=1$ we convene that $1^{*}=\infty$ ). We apply the previous estimate with $v:=w^{2}$ : Taking into account that $|m(v)| \lesssim$ $\|w\|_{1}^{2}$ and $\|v\|_{1^{*}}=\|w\|_{q}^{2}$ with $q:=2 \cdot 1^{*}$, we obtain

$$
\|w\|_{q}^{2}=\|v\|_{1^{*}} \lesssim\|\nabla v\|_{1}+|m(v)| \lesssim\|w \nabla w\|_{1}+\|w\|_{1}^{2},
$$

and taking into account the general inequality $\|f g\|_{1} \leq\|f\|_{2}\|g\|_{2}$ we get

$$
\|w\|_{q} \lesssim\left(\|w\|_{2}\|\nabla w\|_{2}+\|w\|_{1}^{2}\right)^{\frac{1}{2}} \leq\|w\|_{2}^{\frac{1}{2}}\|\nabla w\|_{2}^{\frac{1}{2}}+\|w\|_{1} .
$$


We can now prove (4.11). Since $q=2 \cdot 1^{*}$ is always strictly larger than 2 , by Hölder's inquality we have

$$
\|w\|_{2} \leq\|w\|_{1}^{1-\lambda}\|w\|_{q}^{\lambda}
$$

where $\lambda$ solves the equation $\frac{1}{2}=(1-\lambda) \frac{1}{1}+\lambda \frac{1}{q}$; that is,

$$
\lambda=\frac{d}{d+1}
$$

Plugging (4.12) into (4.13) we get

$$
\|w\|_{2} \lesssim\|w\|_{1}^{1-\lambda}\|w\|_{2}^{\frac{\lambda}{2}}\|\nabla w\|_{2}^{\frac{\lambda}{2}}+\|w\|_{1}
$$

and taking into account that $\|w\|_{1} \lesssim\|w\|_{2}$ we finally obtain

$$
\|w\|_{2}^{1-\frac{\lambda}{2}} \lesssim\|w\|_{1}^{1-\lambda}\|\nabla w\|_{2}^{\frac{\lambda}{2}}+\|w\|_{1}^{1-\frac{\lambda}{2}}
$$

This estimate and (4.14) imply (4.11).

Proof of Lemma 3.3. (a) For the duality we have,

$$
\begin{aligned}
& \inf \left\{\int_{R_{L}} \frac{1}{2}|\mathbf{b}|^{2} \mid \mathbf{b} \in L^{2}\left(R_{L}, \mathbb{R}^{n}\right),\right. \\
& \left.\operatorname{div} \mathbf{b}+m \in[-1,1] \text { in } R_{L}, \mathbf{b} \cdot \nu=g \text { on } \partial R_{L}\right\} \\
& =\inf _{u, \mathbf{b}}\left\{\int_{R_{L}} \frac{1}{2}|\mathbf{b}|^{2} \mid \mathbf{b} \in L^{2}\left(R_{L}, \mathbb{R}^{n}\right), u \in L^{\infty}\left(R_{L},[-1,1]\right),\right. \\
& \left.\quad \operatorname{div} \mathbf{b}=u-m \text { in } R_{L}, \mathbf{b} \cdot \nu=g \text { on } \partial R_{L}\right\} \\
& =\inf _{u, \mathbf{b}} \sup _{v}\left\{\int_{R_{L}} \frac{1}{2}|\mathbf{b}|^{2}+(\mathbf{b} \cdot \nabla v+(u-m) v)-\int_{\partial R_{L}} g v\right. \\
& =\sup _{v} \inf _{u, \mathbf{b}}\left\{\int_{R_{L}} \frac{1}{2}|\mathbf{b}|^{2}+(\mathbf{b} \cdot \nabla v+(u-m) v)-\int_{\partial R_{L}} g v\right. \\
& =\sup _{v}\left\{-\int_{R_{L}} \frac{1}{2}|\nabla v|^{2}+(m v+|v|)-\int_{\partial R_{L}} g v \mid v \in H^{1}\left(R_{L}\right)\right\} \\
& =-\inf _{v}\left\{\int_{R_{L}} \frac{1}{2}|\nabla v|^{2}+(m v+|v|)+\int_{\partial R_{L}} g v \mid v \in H^{1}\left(R_{L}\right)\right\} .
\end{aligned}
$$


The passage to line (4.15) follows from the simple observation: For any $f$ defined on $R_{L}$, the supremum of $\int_{R_{L}} f v$ over all $v \in H^{1}\left(R_{L}\right)$ is $+\infty$ unless $f \equiv 0$. In our case $f=u-m-\operatorname{div} \mathbf{b}$. The passage to line (4.16) follows from a min-max theorem (an infinite-dimensional version which fits our needs is given in [3], Chapter I, Proposition 1.1); note that the functional is convex in $(u, \mathbf{b})$ and linear - and therefore concave - in $v$. Line (4.17) follows by noting that for fixed $v$, the minimum is attained when $\mathbf{b}=-\nabla v$ and $u=\operatorname{sign} v$.

We turn to part (b). The trace formula of Lemma 3.2 was stated and proved on a unit cube for convenience. However, one readily notes that the same estimate holds true for a rectangular box $R=(0,1) \times\left(a_{2}, b_{2}\right) \times \cdots \times\left(a_{n}, b_{n}\right)$ provided

$$
\frac{1}{2} \leq b_{2}-a_{2}, \ldots, b_{n}-a_{n} \leq 1
$$

Thus for $v \in H^{1}(R)$ and any $0<\epsilon \leq 1$,

$$
\begin{gathered}
\int_{\{0\} \times\left(a_{2}, b_{2}\right) \times \cdots \times\left(a_{n}, b_{n}\right)}|v|^{2} d x^{\prime} \\
\lesssim \epsilon \int_{R}|\nabla v|^{2} d x+\left(\frac{1}{\epsilon^{(n+1) / 2}} \int_{R}|v| d x\right)^{2}
\end{gathered}
$$

The same application of Lemma 3.2 yields an analogous inequality with respect to the $i$-th faces of a rectangular box $R=\left(a_{1}, b_{1}\right) \times \cdots \times\left(a_{i-1}, b_{i-1}\right) \times(0,1) \times$ $\left(a_{i+1}, b_{i+1}\right) \times \cdots \times\left(a_{n}, b_{n}\right)$. Now let $R_{L}$ be a rectangular box satisfying (2.2) and let $L_{i}:=b_{i}-a_{i}$. For each of the $i$-th faces, we may rescale the appropriate inequality in $x_{i}$ by $L_{i}$. Then adding the respective terms in the inequalities for all the faces of $\partial R_{L}$, taking the square root of both sides of the inequality, and using (2.2) again, we obtain for any $v \in H^{1}\left(R_{L}\right)$ and $0<\epsilon \leq 1$

$$
\begin{aligned}
& \left(\int_{\partial R_{L}}|v|^{2} d x^{\prime}\right)^{1 / 2} \\
& \quad \leq C\left((\epsilon L)^{1 / 2}\left(\int_{R_{L}}|\nabla v|^{2} d x\right)^{1 / 2}+\frac{1}{(\epsilon L)^{(n+1) / 2}} \int_{R_{L}}|v| d x\right)
\end{aligned}
$$

for some constant $C$, fixed for the remainder of the proof.

Let $\epsilon>0$ be defined as

$$
\begin{gathered}
\epsilon:=\frac{1}{L}\left(\int_{\partial R_{L}} g^{2}\right)^{\frac{1}{n+1}}\left(\frac{C}{1-|m|}\right)^{\frac{2}{n+1}} \\
\text { i.e., } \quad(\epsilon L)^{\frac{n+1}{2}}=\frac{C}{1-|m|}\left(\int_{\partial R_{L}} g^{2}\right)^{\frac{1}{2}} .
\end{gathered}
$$


Note that by (3.3), $\epsilon \leq 1$ for a suitable choice of $C_{0}$. By the application of Lemma 3.2 in the form of (4.18), we have for any $v \in H^{1}\left(R_{L}\right)$,

$$
\begin{aligned}
\int_{R_{L}} & \frac{1}{2}|\nabla v|^{2}+\int_{R_{L}}(m v+|v|)+\int_{\partial R_{L}} g v \\
\geq & \int_{R_{L}} \frac{1}{2}|\nabla v|^{2}+(1-|m|) \int_{R_{L}}|v|+\int_{\partial R_{L}} g v \\
\geq & \int_{R_{L}} \frac{1}{2}|\nabla v|^{2}+(1-|m|) \int_{R_{L}}|v|-\left(\int_{\partial R_{L}} g^{2}\right)^{\frac{1}{2}}\left(\int_{\partial R_{L}} v^{2}\right)^{\frac{1}{2}} \\
\geq & \int_{R_{L}} \frac{1}{2}|\nabla v|^{2}+(1-|m|) \\
& \times \int_{R_{L}}|v|-C\left(\int_{\partial R_{L}} g^{2}\right)^{\frac{1}{2}}(\epsilon L)^{\frac{1}{2}}\left(\int_{R_{L}}|\nabla v|^{2}\right)^{\frac{1}{2}} \\
\geq & -\frac{\epsilon L C^{2}}{2} \int_{\partial R_{L}} g^{2}+\left((1-|m|)-\frac{C}{(\epsilon L)^{\frac{n+1}{2}}}\left(\int_{\partial R_{L}} g^{2}\right)^{\frac{1}{2}} \frac{1}{(\epsilon L)^{\frac{n+1}{2}}} \int_{R_{L}}|v|\right. \\
= & -\frac{C^{\frac{2 n+4}{n+1}}}{2(1-|m|)^{\frac{2}{n+1}}}\left(\int_{\partial R_{L}} g^{2}\right)^{\frac{n+2}{n+1}} \cdot \\
& .
\end{aligned}
$$

Here we used the Cauchy-Schwarz inequality in (4.20) and the trivial inequality $\frac{1}{2} a^{2}-a b \geq-\frac{1}{2} b^{2}$ for (4.21). Part (b) of Lemma 3.3 now follows from (3.2) and (4.22), with a modified choice of $C_{0}$ to accommodate both (4.22) and $\epsilon \leq 1$ in (4.19).

Proof of Lemma 3.4, Let $g \in L^{2}\left(\partial R_{L}\right)$ satisfy (3.3) and let $\tilde{\mathbf{b}}$ be a minimizer of the (2.4). By Lemma 3.3 .

$$
\int_{R_{L}}|\tilde{\mathbf{b}}|^{2} \leq C_{0}\left(\int_{\partial R_{L}} g^{2}\right)^{\frac{n+2}{n+1}}
$$

where $C_{0}$ is as in Lemma 3.3. By definition there exists $\tilde{u} \in L^{\infty}\left(R_{L},[-1,1]\right)$ such that $\operatorname{div} \tilde{\mathbf{b}}+m=\tilde{u}$, and $\tilde{\mathbf{b}} \cdot \nu=g$ on $\partial R_{L}$. By (2.2) we may divide $R_{L}$ up into boxes $R_{i}$ whose side lengths are all between $1 / 2$ and 1 . Note that the number of $R_{i}$ is bounded above by $2^{n} L^{n}$. On each $R_{i}$ we may choose $u_{i} \in B V\left(R_{i},\{ \pm 1\}\right)$ such that

$$
\int_{R_{i}} u_{i}=\int_{R_{i}} \tilde{u}
$$

where $u_{i}$ has exactly one interface of the form $x_{1}=\alpha$. We may now solve

$$
\left\{\begin{array}{cl}
-\triangle v_{i}=u_{i}-\tilde{u} & \text { in } R_{i} \\
\nabla v_{i} \cdot \nu=0 & \text { on } \partial R_{i} .
\end{array}\right.
$$

Setting $\mathbf{b}_{i}=\tilde{\mathbf{b}}-\nabla v_{i}$, we find

$$
\operatorname{div} \mathbf{b}_{i}=\operatorname{div} \tilde{\mathbf{b}}+u_{i}-\tilde{u}=\tilde{u}-m+u_{i}-\tilde{u}=u_{i}-m \quad \text { on } R_{i} .
$$


On the other hand, we have

$$
\int_{R_{i}}\left|\nabla v_{i}\right|^{2}=\int_{R_{i}}\left(-\triangle v_{i}\right) v_{i}=\int_{R_{i}}\left(u_{i}-\tilde{u}\right) v_{i} \leq\left(\int_{R_{i}}\left(u_{i}-\tilde{u}\right)^{2}\right)^{\frac{1}{2}}\left(\int_{R_{i}} v_{i}^{2}\right)^{\frac{1}{2}} .
$$

Since the rectangle $R_{i}$ has side lengths between $1 / 2$ and 1, Poincaré's inequality (for functions with zero mean) gives

$$
\left(\int_{R_{i}} v_{i}^{2}\right)^{\frac{1}{2}} \lesssim\left(\int_{R_{i}}\left|\nabla v_{i}\right|^{2}\right)^{\frac{1}{2}}
$$

which combined with (4.25) yields

$$
\int_{R_{i}}\left|\nabla v_{i}\right|^{2} \lesssim \int_{R_{i}}\left|u_{i}-\tilde{u}\right|^{2} \lesssim\left|R_{i}\right| \lesssim 1
$$

Thus defining $(u, \mathbf{b})$ on $R_{L}$ to be $\left(u_{i}, \mathbf{b}_{i}\right)$ on $R_{i}$, we find

$$
\operatorname{div} \mathbf{b}=u-m \quad \text { on } R_{L}, \quad \mathbf{b} \cdot \nu=g \quad \text { on } \partial R_{L} .
$$

Note that the additional interfacial area introduced by piecing together the $u_{i}$ is bounded by $2 n L^{n}$. Thus using (4.23),

$$
\begin{aligned}
E\left(\mathbf{b}, u, R_{L}\right) & \lesssim \sum_{i}\left(\frac{1}{2} \int_{R_{i}}\left|\mathbf{b}_{i}\right|^{2}+\int_{R_{i}}\left|\nabla u_{i}\right|\right)+L^{n} \\
& \lesssim \frac{1}{2} \int_{R_{L}}|\tilde{\mathbf{b}}|^{2}+\sum_{i}\left(\frac{1}{2} \int_{R_{i}}\left|\nabla v_{i}\right|^{2}+\int_{R_{i}}\left|\nabla u_{i}\right|\right)+L^{n} \\
& \lesssim\left(\int_{\partial R_{L}} g^{2}\right)^{\frac{n+2}{n+1}}+L^{n}+L^{n}+L^{n}
\end{aligned}
$$

Finally,

$$
\sigma^{g}(L) \leq \frac{1}{L^{n}} E\left(\mathbf{b}, u, R_{L}\right) \leq C\left(1+\frac{1}{L^{n}}\left(\int_{\partial R_{L}} g^{2}\right)^{\frac{n+2}{n+1}}\right),
$$

for some constant $C$. Lemma 3.4 now follows with $C_{1}:=\max \left\{C, C_{0}\right\}$.

Proof of Lemma 3.5. We give the proof for minimizers over $\mathcal{A}\left(Q_{L}\right)$ and $\mathcal{A}_{0}\left(Q_{L}\right)$ simultaneously. Let $\left(u_{0}, \mathbf{b}_{0}\right)$ be a minimizer of $E\left(u_{0}, \mathbf{b}_{0}, Q_{L}\right)$ on either $\mathcal{A}\left(Q_{L}\right)$ or $\mathcal{A}_{0}\left(Q_{L}\right)$, and let $a \in Q_{L}$. For $l<L$ we define

$$
h(l):=E\left(u_{0}, \mathbf{b}_{0}, \overline{Q_{l}(a)} \cap Q_{L}\right) .
$$

Note that evaluating the energy on $\overline{Q_{l}(a)} \cap Q_{L}$ takes into account the part of the measure $\left|\nabla u_{0}\right|$ concentrated on the surface $\partial Q_{l}(a) \cap Q_{L}$. In particular, $h(\cdot)$ is an upper semi-continuous, nondecreasing function.

By Lemma 3.4 applied to $g:=0$,

$$
h(L) \leq E\left(u_{0}, \mathbf{b}_{0}, Q_{L}\right)=\sigma\left(Q_{L}\right) L^{n} \leq \sigma^{0}\left(Q_{L}\right) L^{n} \leq C_{1} L^{n},
$$

for the case of minimizers over $\mathcal{A}\left(Q_{L}\right)$. The same holds true for minimizers over $\mathcal{A}_{0}\left(Q_{L}\right)$ provided we remove the third term in this chain of (in)equalities.

We may assume that there exists an $l<L$ such that

$$
h(l) \geq 3 C_{1} l^{n} ;
$$


otherwise there is nothing to prove. Consider the subset of $\mathbb{R}$,

$$
S:=\left\{l \in[0, L] \mid h(l) \geq 3 C_{1} l^{n}\right\} .
$$

Since $h$ is upper semi-continuous, $S$ is closed and, in view of (4.27), nonempty. Hence

$$
l_{*}:=\sup S
$$

belongs to $S$, and by (4.26),$l_{*}<L$. Thus we have

$$
h\left(l_{*}\right) \geq 3 C_{1} l_{*}^{n} \quad \text { and } \quad h(l)<3 C_{1} l^{n} \quad \forall l \in\left(l_{*}, L\right] .
$$

Since $h$ is nondecreasing, we must have

$$
h\left(l_{*}\right)=3 C_{1} l_{*}^{n}
$$

and

$$
h^{\prime}\left(l_{*}\right):=\limsup _{\epsilon \rightarrow 0^{+}} \frac{h\left(l_{*}+\epsilon\right)-h\left(l_{*}\right)}{\epsilon} \leq 3 n C_{1} l_{*}^{n-1} .
$$

Our goal is now to show that $l_{*}$ must be bounded above independently of $L$. To this end, without loss of generality we may assume

$$
l_{*} \geq \max \left\{(3 n)^{1 / 2} C_{1}, \frac{8 n}{C_{1}}\right\} .
$$

For all $\epsilon>0$ we have, by definition of $h$

$$
\frac{1}{\epsilon} \int_{\left(Q_{l_{*}+\epsilon}(a)-Q_{l_{*}}(a)\right) \cap Q_{L}} \frac{1}{2}\left|\mathbf{b}_{0}\right|^{2} \leq \frac{h\left(l_{*}+\epsilon\right)-h\left(l_{*}\right)}{\epsilon} .
$$

Next we take the $\lim \sup$ as $\epsilon \rightarrow 0^{+}$in (4.31). To pass to the limit in the integral on the left-hand side we need, for instance, that $\mathbf{b}_{0}$ is continuous. This follows by standard elliptic regularity: Indeed, since

$$
\mathbf{b}_{0}=-\nabla v, \quad \operatorname{div} \mathbf{b}_{0}=u_{0}-m \text { in } Q_{L},
$$

with $v=0$ on $\partial Q_{L}$ in the case of $\mathcal{A}\left(Q_{L}\right)$, and $\nabla v \cdot \nu=0$ on $\partial Q_{L}$ in the case of $\mathcal{A}_{0}\left(Q_{L}\right)$, standard Schauder theory implies

$$
v \in C^{1, \alpha}\left(\bar{Q}_{L}\right), \quad \text { and hence } \quad \mathbf{b}_{0} \in C^{0, \alpha}\left(\bar{Q}_{L}\right),
$$

for all $\alpha<1$. Thus we deduce from (4.29), (4.30), and (4.31) that

$$
\int_{\partial Q_{l_{*}}(a) \cap Q_{L}} \frac{1}{2}\left(\mathbf{b}_{0} \cdot \nu\right)^{2} \leq \int_{\partial Q_{l_{*}}(a) \cap Q_{L}} \frac{1}{2}\left|\mathbf{b}_{0}\right|^{2} \leq 3 n C_{1} l_{*}^{n-1} \leq \frac{1}{C_{1}} l_{*}^{n+1} .
$$

Notice that the rectangular box $Q_{l_{*}}(a) \cap Q_{L}$ is not too anisotropic in the sense that it satisfies (2.2) with $L$ replaced by $l_{*}$. Also note that

$$
\partial\left(Q_{l_{*}}(a) \cap Q_{L}\right)=\left(\partial Q_{l_{*}}(a) \cap Q_{L}\right) \cup\left(\overline{Q_{l_{*}}(a)} \cap \partial Q_{L}\right) .
$$

Hence by (4.32), we may apply Lemma 3.4 to

$$
g:=\left\{\begin{array}{cc}
\mathbf{b}_{0} \cdot \nu & \text { on } \partial Q_{l_{*}}(a) \cap Q_{L}, \\
0 & \text { on } \overline{Q_{l_{*}}(a)} \cap \partial Q_{L}
\end{array}\right.
$$



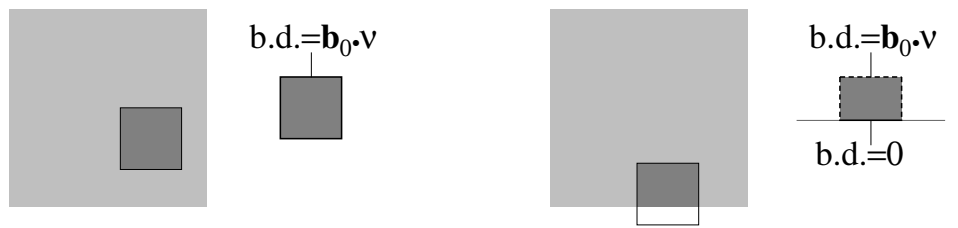

$Q_{L} \quad Q_{l_{*}}(a) \cap Q_{L}$

Figure 1. Two possible positions for $Q_{l_{*}}(a) \cap Q_{L}$, and corresponding Neumann boundary conditions on $\partial\left(Q_{l_{*}}(a) \cap Q_{L}\right)$.

(see Figure 11) and obtain

$$
\sigma^{g}\left(Q_{l_{*}}(a) \cap Q_{L}\right) \leq C_{1}\left(1+\frac{1}{l_{*}^{n}}\left(\int_{\partial\left(Q_{l_{*}}(a) \cap Q_{L}\right)} \frac{1}{2} g^{2}\right)^{\frac{n+2}{n+1}}\right) .
$$

Hence there exists $\left(u^{*}, \mathbf{b}^{*}\right)$ on $Q_{l_{*}}(a) \cap Q_{L}$ with

$$
\mathbf{b}^{*} \cdot \nu=0 \quad \text { on } \overline{Q_{l_{*}}(a)} \cap \partial Q_{L} \quad \text { and } \quad \mathbf{b}^{*} \cdot \nu=\mathbf{b}_{0} \cdot \nu \text { on } \partial Q_{l_{*}}(a) \cap Q_{L},
$$
and

$$
\begin{aligned}
E\left(u^{*}, \mathbf{b}^{*}, Q_{l_{*}}(a) \cap Q_{L}\right) & \leq C_{1}\left(l_{*}^{n}+\left(\int_{\partial Q_{l_{*}}(a) \cap Q_{L}} \frac{1}{2}\left(\mathbf{b}_{0} \cdot \nu\right)^{2}\right)^{\frac{n+2}{n+1}}\right) \\
& \leq C_{1}\left(l_{*}^{n}+\left(3 n C_{1} l_{*}^{n-1}\right)^{\frac{n+2}{n+1}}\right) .
\end{aligned}
$$

By (4.33),

$$
(\tilde{u}, \tilde{\mathbf{b}}):= \begin{cases}\left(u^{*}, \mathbf{b}^{*}\right) & \text { on } Q_{l_{*}}(a) \cap Q_{L}, \\ \left(u_{0}, \mathbf{b}_{0}\right) & \text { on } Q_{L} \backslash Q_{l_{*}}(a)\end{cases}
$$

is admissible on $\mathcal{A}$ and $\mathcal{A}_{0}$ respectively. Hence in either case,

$$
\begin{aligned}
E\left(u_{0}, \mathbf{b}_{0}, Q_{l_{*}}(a) \cap Q_{L}\right)+E\left(u_{0}, \mathbf{b}_{0}, Q_{L} \backslash Q_{l_{*}}(a)\right) \\
\leq E\left(u_{0}, \mathbf{b}_{0}, Q_{L}\right) \\
\leq E\left(\tilde{u}, \tilde{\mathbf{b}}, Q_{L}\right) \\
\leq E\left(u^{*}, \mathbf{b}^{*}, Q_{l_{*}}(a) \cap Q_{L}\right)+E\left(u_{0}, \mathbf{b}_{0}, Q_{L} \backslash Q_{l_{*}}(a)\right)+4 n l_{*}^{n-1} .
\end{aligned}
$$

Thus

$$
\begin{aligned}
h\left(l_{*}\right) \leq & \leq\left(u_{0}, \mathbf{b}_{0}, Q_{l_{*}}(a) \cap Q_{L}\right)+4 n l_{*}^{n-1} \\
& \leq\left(u^{*}, \mathbf{b}^{*}, Q_{l_{*}}(a) \cap Q_{L}\right)+8 n l_{*}^{n-1} \\
\leq & C_{1}\left(l_{*}^{n}+\left(3 n C_{1} l_{*}^{n-1}\right)^{\frac{n+2}{n+1}}\right)+8 n l_{*}^{n-1} \\
\underset{4.340}{\leq} & 2 C_{1} l_{*}^{n}+C_{1}\left(3 n C_{1} l_{*}^{n-1}\right)^{\frac{n+2}{n+1}} .
\end{aligned}
$$

Combining the above with (4.28), we find

$$
3 C_{1} l_{*}^{n} \leq 2 C_{1} l_{*}^{n}+C_{1}\left(3 n C_{1} l_{*}^{n-1}\right)^{\frac{n+2}{n+1}},
$$


SO

$$
l_{*}^{n} \leq\left(3 n C_{1} l_{*}^{n-1}\right)^{\frac{n+2}{n+1}}, \quad \text { i.e., }, \quad l_{*} \leq\left(3 n C_{1}\right)^{\frac{n+1}{2}} .
$$

Hence the lemma follows for

$$
C_{2}:=\max \left\{(3 n)^{1 / 2} C_{1}, \frac{8 n}{C_{1}},\left(3 n C_{1}\right)^{\frac{n+1}{2}}\right\} .
$$

Proof of Lemma 3.6. As in Lemma 3.5, we present the proof for a minimizer $\left(u_{0}, \mathbf{b}_{0}\right)$ of $E\left(u, \mathbf{b}, Q_{L}\right)$ over $\mathcal{A}\left(Q_{L}\right)$ and over $\mathcal{A}_{0}\left(Q_{L}\right)$ simultaneously. Since here there is no need to keep track of constants, we adopt the $\lesssim$ convention.

The crucial ingredient here is Lemma 3.5, which yields a uniform bound on local averages of $\left|\mathbf{b}_{0}\right|^{2}$; that is,

$$
\int_{Q_{1}(a)}\left|\mathbf{b}_{0}\right|^{2} \lesssim 1
$$

for every cube $Q_{1}(a)$ contained in $Q_{L}$.

The rest of the proof relies on a standard argument of elliptic regularity theory and is briefly sketched. First of all, we recall that for every $p$ with $1<p<\infty$ the norm

$$
\|\triangle v\|_{p}+\|\nabla v\|_{p}+\|v\|_{p}
$$

is equivalent to the standard norm on the Sobolev space $W^{2, p}\left(Q_{1}\right)$. Then a standard compactness argument shows that the same holds true for

$$
\|\triangle v\|_{p}+\|\nabla v\|_{1}+|m(v)|
$$

where $m(v)$ denotes the average of $v$ on $Q_{1}$. Since $W^{2,2 n}\left(Q_{1}\right)$ is embedded in $W^{1, \infty}\left(Q_{1}\right)$, we have

$$
\|v\|_{W^{1, \infty}} \lesssim\|v\|_{W^{2,2 n}} \lesssim\|\triangle v\|_{2 n}+\|\nabla v\|_{1}+|m(v)|
$$

for every $v \in W^{2,2 n}\left(Q_{1}\right)$, and by density also for every $v \in W^{1,1}\left(Q_{1}\right)$.

In order to apply estimate (4.36) to $\mathbf{b}_{0}$ we recall that there exists $v \in H^{1}\left(Q_{L}\right)$ such that $\mathbf{b}=-\nabla v$ in $Q_{L}$. Fix a cube $Q_{1}(a)$ contained in $Q_{L}$ : Upon addition of a constant we can assume that the average of $v$ on $Q_{1}(a)$ vanishes. Hence (4.35), (4.36), and the fact that $\triangle v=\operatorname{div} \mathbf{b}=u-m$ yield

$$
\left\|\mathbf{b}_{0}\right\|_{\infty}=\|\nabla v\|_{\infty} \lesssim\|\triangle v\|_{2 n}+\|\nabla v\|_{1}+|m(v)|=\|u-m\|_{2 n}+\left\|b_{0}\right\|_{1} \lesssim 1
$$

where all norms refer to the domain $Q_{1}(a)$. The $L^{\infty}$-bound for $\mathbf{b}_{0}$ on the whole $Q_{L}$ is obtained by covering this cube by cubes of the form $Q_{1}(a)$.

Proof of Lemma 3.7. The proofs of statements (i) and (ii) are very similar. We present (ii). Let $(u, \mathbf{b})$ be a minimizer for $E$ on $\mathcal{A}^{g}\left(Q_{L}\right)$. Let $C_{3}$ denote a constant which we will choose shortly. Since $L \geq 2 C_{3} \Lambda$, the frame $Q_{L+2 C_{3} \Lambda} \backslash Q_{L}$ can be partitioned into rectangular boxes $R_{i}$ of side lengths between $\frac{1}{2} C_{3} \Lambda$ and $C_{3} \Lambda$ (see Figure 2).

We now apply Lemma 3.4 to any of these $R_{i}$ with $C_{3} \Lambda$ playing the role of $L$ and with boundary data

$$
\left\{\begin{array}{cc}
g & \text { on } \partial R_{i} \cap \partial Q_{L} \\
0 & \text { on } \partial R_{i} \backslash \overline{Q_{L}}
\end{array}\right.
$$




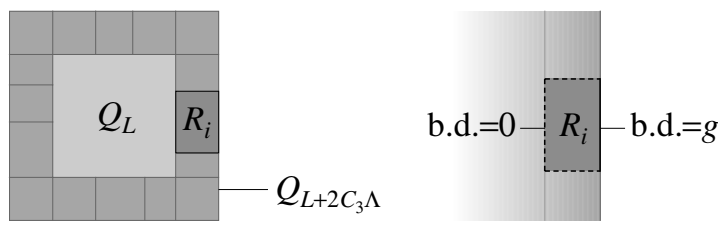

Figure 2. Left: Frame $Q_{L+2 C_{3} \Lambda} \backslash Q_{L}$ partitioned into rectangular boxes $R_{i}$ of side lengths between $\frac{1}{2} C_{3} \Lambda$ and $C_{3} \Lambda$. Right: Boundary values on $\partial R_{i}$ for the application of Lemma 3.4

The assumption on the boundary data in Lemma 3.4 becomes

$$
\int_{\partial R_{i} \cap \partial Q_{L}} g^{2} \leq \frac{1}{C_{1}}\left(C_{3} \Lambda\right)^{n+1},
$$

where $C_{1}$ is the constant from Lemma 3.4. Since $\sup |g|=\Lambda$, it is satisfied by choosing

$$
C_{3}:=\sqrt{2 n C_{1}} .
$$

Thus Lemma 3.4 yields $\left(u_{i}, \mathbf{b}_{i}\right)$ on $R_{i}$ such that

$$
\begin{aligned}
E\left(u_{i}, \mathbf{b}_{i}, R_{i}\right) & \leq C_{1}\left(\left(C_{3} \Lambda\right)^{n}+\left(\int_{\partial R_{i} \cap \partial Q_{L}} g^{2}\right)^{\frac{n+2}{n+1}}\right) \\
& \leq C \Lambda^{n}\left(1+\Lambda^{2}\right)
\end{aligned}
$$

and

$$
\mathbf{b}_{i} \cdot \nu=\left\{\begin{array}{cc}
g=\mathbf{b} \cdot \nu & \text { on } \partial R_{i} \cap \partial Q_{L} \\
0 & \text { on } \partial R_{i} \backslash \overline{Q_{L}}
\end{array}\right.
$$

Thus the pair

$$
\left(u_{*}, \mathbf{b}_{*}\right):=\left\{\begin{array}{cc}
(u, \mathbf{b}) & \text { on } Q_{L}, \\
\left(u_{i}, \mathbf{b}_{i}\right) & \text { on } \partial R_{i}
\end{array}\right.
$$

is admissible for $\sigma^{0}\left(Q_{L+2 C_{3} \Lambda}\right)$, and hence

$$
\begin{aligned}
\left(L+2 C_{3} \Lambda\right)^{n} \sigma^{0}\left(Q_{L+2 C_{3} \Lambda}\right) & \leq E\left(u_{*}, \mathbf{b}_{*}, Q_{L+2 C_{3} \Lambda}\right) \\
& \leq E\left(u, \mathbf{b}, Q_{L}\right)+\sum_{i}\left(E\left(u_{i}, \mathbf{b}_{i}, R_{i}\right)+4 n\left(C_{3} \Lambda\right)^{n-1}\right) \\
& \leq L^{n} \sigma^{g}\left(Q_{L}\right)+C\left(\frac{L}{\Lambda}\right)^{n-1}\left(\Lambda^{n}\left(1+\Lambda^{2}\right)+\Lambda^{n-1}\right) .
\end{aligned}
$$

In the last line above, we used the fact that the number of $R_{i}$ does not exceed $(L / \Lambda)^{n-1}$. Thus

$$
\sigma^{0}\left(Q_{L+2 C_{3} \Lambda}\right) \leq \sigma^{g}\left(Q_{L}\right)+\frac{C\left(1+\Lambda+\Lambda^{3}\right)}{L} \leq \sigma^{g}\left(Q_{L}\right)+\frac{C\left(1+\Lambda^{3}\right)}{L} .
$$

The proof of (i) is very similar, with the reverse roles in both the frame and boundary data of Figure 2, see Figure 3 . 

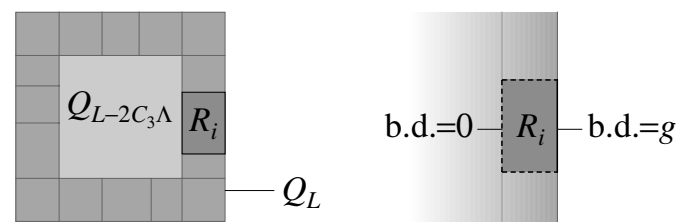

Figure 3. The analogous structure for the proof of statement (i)

Proof of Lemma 3.8. Let $\left(u_{0}, \mathbf{b}_{0}\right)$ be a minimizer of $E$ on $\mathcal{A}\left(Q_{L}\right)$. Trivially, $\left(u_{0}, \mathbf{b}_{0}\right)$ is also a minimizer for $\sigma^{g}\left(Q_{L}\right)$ with

$$
g=\mathbf{b}_{0} \cdot \nu \quad \text { on } \partial Q_{L} .
$$

Hence

$$
\sigma\left(Q_{L}\right)=\sigma^{g}\left(Q_{L}\right) .
$$

By Lemma 3.6, we have

$$
|g| \lesssim 1
$$

By Lemma 3.7(ii), there exist constants $C_{4}, C$ such that

$$
\sigma^{0}\left(Q_{L+C_{4}}\right) \leq \sigma^{g}\left(Q_{L}\right)+\frac{C}{L}
$$

provided $L \geq C_{4}$. Combining the above with (4.39) gives the result.

Proof of Lemma 3.9. Let $\left(u_{0}, \mathbf{b}_{0}\right)$ be a minimizer of $E$ on $\mathcal{A}_{0}$. Let $Q_{l}(a) \subset Q_{L}$ and let $\left(u_{*}, \mathbf{b}_{*}\right)$ be a minimizer for $\sigma^{g}\left(Q_{l}(a)\right)$ where

$$
g= \begin{cases}\mathbf{b}_{0} \cdot \nu & \text { on } \partial Q_{l}(a) \cap Q_{L}, \\ 0 & \text { on } \partial Q_{L} \cap \overline{Q_{l}(a)} .\end{cases}
$$

Thus $(\tilde{u}, \tilde{\mathbf{b}})$ defined by

$$
(\tilde{u}, \tilde{\mathbf{b}}):= \begin{cases}\left(u_{*}, \mathbf{b}_{*}\right) & \text { on } Q_{l}(a), \\ \left(u_{0}, \mathbf{b}_{0}\right) & \text { on } Q_{L} \backslash Q_{l}(a)\end{cases}
$$

is admissible for $\sigma^{0}\left(Q_{L}\right)$. Thus

$$
E\left(u_{0}, \mathbf{b}_{0}, Q_{L}\right) \leq E\left(\tilde{u}, \tilde{\mathbf{b}}, Q_{L}\right) .
$$

By construction, we have

$$
E\left(u_{0}, \mathbf{b}_{0}, Q_{l}(a)\right) \leq E\left(u_{*}, \mathbf{b}_{*}, Q_{l}(a)\right)+4 n l^{n-1}
$$

or

$$
\frac{1}{l^{n}} E\left(u_{0}, \mathbf{b}_{0}, Q_{l}(a)\right) \leq \sigma^{g}\left(Q_{l}(a)\right)+\frac{C}{l},
$$

for some constant $C$. Lemma 3.6 implies that

$$
|g| \lesssim 1 \quad \text { on } \partial Q_{l}(a) \cap Q_{L},
$$

and hence by Lemma 3.7(i) there exist constants $C_{5}, C$ such that

$$
\sigma^{g}\left(Q_{l}(a)\right) \leq \sigma^{0}\left(Q_{l-C_{5}}\right)+\frac{C}{l},
$$

for $l \geq C_{5}$. This combined with (4.40) yields the lemma. 
Proof of Lemma 3.10, Let

$$
\sigma_{+}^{*}:=\liminf _{l \rightarrow \infty} \sigma^{0}\left(Q_{l}\right) \quad \text { and } \quad \sigma_{-}^{*}:=\limsup _{l \rightarrow \infty} \sigma\left(Q_{l}\right) .
$$

Notice that from Lemma 3.1 we have the following results: Statements (iv) and (v) respectively imply

$$
\sigma_{+}^{*}<\infty \quad \text { and } \quad \sigma_{-}^{*}>0 ;
$$

statement (i) gives

$$
\sigma_{-}^{*} \leq \sigma_{+}^{*} ;
$$

and from statements (ii) and (iii) respectively we have for all $l \geq C$,

$$
\sigma\left(Q_{l}\right) \leq \limsup _{k \rightarrow \infty, k \in \mathbb{N}} \sigma\left(Q_{k l}\right) \leq \sigma_{-}^{*}
$$

and

$$
\sigma^{0}\left(Q_{l}\right) \geq \liminf _{k \rightarrow \infty, k \in \mathbb{N}} \sigma^{0}\left(Q_{k l}\right)-\frac{C}{l} \geq \sigma_{+}^{*}-\frac{C}{l} .
$$

On the other hand, Lemma 3.8 implies that

$$
\sigma^{0}\left(Q_{l+C_{4}}\right) \leq \sigma\left(Q_{l}\right)+\frac{C}{l} \quad \forall l \geq C_{4} .
$$

Thus for any $l \geq C_{4}$, we have

$$
\sigma_{+}^{*} \underset{(4.44)}{\leq} \sigma^{0}\left(Q_{l+C_{4}}\right)+\frac{C}{l+C_{4}} \underset{(4.45)}{\leq} \sigma\left(Q_{l}\right)+\frac{C}{l} \underset{[4.43)}{\leq} \sigma_{-}^{*}+\frac{C}{l} .
$$

Letting $l$ tend to infinity yields $\sigma_{+}^{*} \leq \sigma_{-}^{*}$, which, combined with (4.42), yields equality. Let

$$
\sigma^{*}:=\sigma_{+}^{*}=\sigma_{-}^{*} .
$$

By (4.41), $\sigma^{*} \in(0, \infty)$.

Finally, we have for all $l \geq C_{4}$,

$$
\sigma\left(Q_{l}\right) \underset{(4.45)}{\geq} \sigma^{0}\left(Q_{l+C_{4}}\right)-\frac{C}{l} \underset{(4.44),(4.46)}{\geq} \sigma^{*}-\frac{C}{l+C_{4}}-\frac{C}{l} .
$$

On the other hand, for $l \geq 2 C_{4}$,

$$
\sigma^{0}\left(Q_{l}\right) \underset{(4.45)}{\leq} \sigma\left(Q_{l-C_{4}}\right)+\frac{C}{l-C_{4}} \underset{(4.43),(4.46)}{\leq} \sigma^{*}+\frac{C}{l-C_{4}} .
$$

This combined with Lemma 3.1(i) concludes the proof.

\section{The Proof of Theorem 1.3}

The proof will follow from Theorem 1.1, the $L^{\infty}$ bound for minimizing $\mathbf{b}$ (Lemma (3.6), and a suitable weak formulation of the Euler-Lagrange equation. Under a regularity assumption for the interface associated with $u$, the Euler-Lagrange equation reduces simply to an equation relating the mean curvature of this interface to the potential $v$ associated with $\mathbf{b}(c f$. [10]). In Lemma [5.1, we give a weak version of this statement which holds regardless of the regularity of the interface associated with $u$.

Throughout this section, given a function $u \in B V\left(Q_{L}, \pm 1\right)$, we denote by $\mu$ the density of the vector-valued measure $\nabla u$ with respect to its total variation $|\nabla u|$. We 
recall that this unit vector field corresponds to the measure-theoretic inner normal of the set of finite perimeter $A:=\{u=1\}$ (cf. [13, Chapter 3).

Lemma 5.1. Let $\left(u_{0}, \mathbf{b}_{0}\right)$ be a minimizer of $E$ on $\mathcal{A}_{0}\left(Q_{L}\right)$. Then there exists $v_{0} \in C^{1}\left(\overline{Q_{L}}\right)$ such that $\mathbf{b}_{0}=-\nabla v_{0}$ and

$$
\int_{Q_{L}}\left(v_{0} \zeta \cdot \mu\right)\left|\nabla u_{0}\right|+\int_{Q_{L}}(\operatorname{div} \zeta-\mu \cdot(D \zeta \mu))\left|\nabla u_{0}\right|=0,
$$

for any smooth vector field $\zeta$ with compact support in $Q_{L}$. Here $D \zeta$ denotes the matrix of partial derivatives $\partial_{j} \zeta_{i}$ and $\mu$ denotes density of $\nabla u_{0}$ with respect to $\left|\nabla u_{0}\right|$.

Note that, while we only need the result for $\zeta$ with compact support, Lemma 5.1 also holds for all smooth vector fields $\zeta$ on $\overline{Q_{L}}$ with $\zeta \cdot \nu=0$ on $\partial Q_{L}$.

Proof of Lemma 5.1. The existence of a potential $v_{0}$ associated to $\mathbf{b}_{0}$ was already established in the Introduction, while the $C^{1}\left(\overline{Q_{L}}\right)$ regularity is a by-product of Lemma 3.6. Let $A_{0}:=\left\{u_{0}=1\right\}$ and let $\zeta: Q_{L} \rightarrow \mathbb{R}^{n}$ be a compactly supported smooth vector field. Note that any admissible $u$ for $\mathcal{A}_{0}\left(Q_{L}\right)$ must have average $m$; alternatively the set $\{u=1\}$ must have volume fraction $(1+m) / 2$. Thus the optimal $u_{0}$ is a mass constrained minimizer, and this must be taken into account when computing the variation of $E$ associated to a vector field $\zeta$.

The assumption $m \in(-1,1)$ implies $0<\left|A_{0}\right|<\left|Q_{L}\right|$, and hence there exists a vector field $\widehat{\zeta}$, compactly supported in $Q_{L}$, such that

$$
0 \neq \int_{A_{0}} \operatorname{div} \widehat{\zeta} d x=\frac{1}{2} \int_{Q_{L}} u_{0} \operatorname{div} \widehat{\zeta} d x .
$$

We now consider the flow $F_{t, s}: Q_{L} \rightarrow Q_{L}$ defined by

$$
F_{t, s}(x):=x+t \zeta(x)+s \widehat{\zeta}(x),
$$

for $t, s$ sufficiently small. Let $u_{t, s}$ be given by

$$
u_{t, s}:=\left\{\begin{array}{cl}
1 & \text { on } F_{t, s}\left(A_{0}\right) \\
-1 & \text { on } Q_{L} \backslash F_{t, s}\left(A_{0}\right) .
\end{array}\right.
$$

Our goal is find $s=\phi(t)$ such that $\left|F_{t, \phi(t)}\left(A_{0}\right)\right|=\left|A_{0}\right|$ for small $t$. To this end, note that the Jacobian of $F_{t, s}$, denoted by $J F_{t, s}$, satisfies ( $c f$. the proof of Theorem 10.4 in $[13$ )

$$
J F_{t, s}=1+t \operatorname{div} \zeta+s \operatorname{div} \widehat{\zeta}+o(t, s)
$$

Thus setting

$$
V(t, s):=\left|F_{t, s}\left(A_{0}\right)\right|=\int_{A_{0}} J F_{t, s} d x,
$$

we have $V(0,0)=(1+m) / 2$ and

$$
\left.\frac{\partial V(t, s)}{\partial s}\right|_{s=t=0}=\int_{A_{0}} \operatorname{div} \widehat{\zeta} d x \neq 0,
$$

and therefore we may apply the implicit function theorem to the equation $V(t, s)=$ $(1+m) / 2$ to obtain a function $s=\phi(t)$ such that $\phi(0)=0$ and

$$
V(t, \phi(t))=\frac{1+m}{2} \text { for small } t .
$$


Note that

$$
\phi^{\prime}(0)=-\frac{\int_{A_{0}} \operatorname{div} \zeta d x}{\int_{A_{0}} \operatorname{div} \widehat{\zeta} d x} .
$$

It follows that the volume of the set $F_{t, \phi(t)}\left(A_{0}\right)$ is equal to the volume of $A_{0}$ for $t$ sufficiently small, which is equivalent to saying that the functions $u_{t, \phi(t)}$ satisfy the mass constraint $\frac{1}{L^{n}} \int_{Q_{L}} u_{t, \phi(t)}=m$ and therefore belong to $\mathcal{A}_{0}\left(Q_{L}\right)$. Let $v_{t}$ denote any potential associated with $u_{t, \phi(t)}$, i.e., $-\triangle v_{t}=u_{t, \phi(t)}-m$ with Neumann boundary conditions. Note that $u_{0,0}=u_{0}$ and $-\nabla v_{0}=\mathbf{b}_{0}$. Hence setting $E_{t}=$ $E\left(u_{t, \phi(t)},-\nabla v_{t, \phi(t)}, Q_{L}\right)$, we must have

$$
\left.\frac{d E_{t}}{d t}\right|_{t=0}=0
$$

To calculate the left-hand side of (5.3), note that this consists of two terms, the surface energy and the nonlocal energy. For the surface energy, the calculation is identical to that of Theorem 10.4 of [13, whereas for the nonlocal part, the calculation is identical to that of Theorem 2.3 of [10]. Together, these calculations yield

$$
\begin{aligned}
\int_{Q_{L}} & {\left[v_{0}\left(\zeta+\phi^{\prime}(0) \widehat{\zeta}\right) \cdot \mu\right]\left|\nabla u_{0}\right| } \\
& +\int_{Q_{L}}\left[\operatorname{div}\left(\zeta+\phi^{\prime}(0) \widehat{\zeta}\right)-\left(D\left(\zeta+\phi^{\prime}(0) \widehat{\zeta}\right) \mu\right) \cdot \mu\right]\left|\nabla u_{0}\right|=0 .
\end{aligned}
$$

Hence

$$
\begin{aligned}
0= & \int_{Q_{L}}\left(v_{0} \zeta \cdot \mu\right)\left|\nabla u_{0}\right|+\int_{Q_{L}}[\operatorname{div} \zeta-\mu \cdot(D \zeta \mu)]\left|\nabla u_{0}\right| \\
& +\phi^{\prime}(0)\left(\int_{Q_{L}}\left(v_{0} \widehat{\zeta} \cdot \mu\right)\left|\nabla u_{0}\right|+\int_{Q_{L}}[\operatorname{div} \widehat{\zeta}-\mu \cdot(D \widehat{\zeta} \mu)]\left|\nabla u_{0}\right|\right) .
\end{aligned}
$$

Thus (5.1) will follow by modifying $v_{0} \in C^{1}\left(\overline{Q_{L}}\right)$ by the addition of a constant so that

$$
\int_{Q_{L}} v_{0} \widehat{\zeta} \cdot \mu\left|\nabla u_{0}\right|+\int_{Q_{L}}[\operatorname{div} \widehat{\zeta}-(D \widehat{\zeta} \mu) \cdot \mu]\left|\nabla u_{0}\right|=0
$$

This is possible because of condition (5.2).

We now prove Theorem 1.3. For convenience of notation, let us break from the tradition of using 0 -subindices for denoting the minimizing pair for $E$ on $\mathcal{A}_{0}\left(Q_{L}\right)$. It will be denoted simply by $(u, \mathbf{b})$.

Proof of Theorem [1.3. The indexed constants $C_{1}$ and $C_{2}$ in this proof will have no relation to those of the previous sections, and note that the generic constant $C$ may change from line to line. Let $(u, \mathbf{b})$ be a minimizer of $E$ on $\mathcal{A}_{0}\left(Q_{L}\right)$ and let $v$ be as in Lemma 5.1. The proof relies on Theorem 1.1 which implies

$$
\int_{B_{R}(a)}|\nabla u| \lesssim R^{n} \quad \text { for all } a \in Q_{L} \text { and } R \geq 1 \text { s.t. } B_{R}(a) \subset Q_{L},
$$

and on Lemma 3.6 which implies

$$
\sup _{Q_{L}}|\nabla v| \lesssim 1
$$


These two bounds will be coupled via Lemma 5.1] with a suitable choice of test function $\zeta$. This test function is a localized piece of the gradient of a mollification of $u$. The localization is centered at $a \in Q_{L}$, and there will be two constant length scales associated with $\zeta$ : A localization length scale $R$ and a mollification length scale $r$. These two scales will be set within the course of the proof. In fact, once constants $C_{1} \geq 1$ and $C_{2} \geq 1$ are chosen, one may choose $R$ and $r$ such that

$$
r \leq \frac{1}{C_{1}} \leq C_{1} \leq 4 C_{2} \leq R .
$$

Fix a smooth cut-off function $\eta(x)$ such that

$$
0 \leq \eta \leq 1, \quad \operatorname{supp} \eta \subset B_{1 / 2}(0), \quad \eta \equiv 1 \text { on } B_{1 / 4}(0) .
$$

For fixed $R$ and $a \in Q_{L}$ such that $B_{R}(a) \subset Q_{L}$, we define

$$
\eta_{R}(x):=\eta\left(\frac{x-a}{R}\right) .
$$

We choose a mollifying kernel $\phi$, supported in $B_{1 / 4}(0)$, and define our test function in (5.1) to be

$$
\zeta:=\eta_{R} \nabla u_{r r}^{* *},
$$

where the mollification $u_{r r}^{* *}$ is defined by (2.1). It is convenient to extend $\zeta, u$, etc. to 0 in the complement of their domains.

We claim that the following estimates hold for the terms in (5.1):

$$
\begin{gathered}
\left|\int_{Q_{L}}(\operatorname{div} \zeta-\mu \cdot(D \zeta \mu))\right| \nabla u|| \lesssim \frac{R^{n}}{r^{2}} \\
\int_{Q_{L}}|\zeta \cdot \mu||\nabla u| \lesssim \frac{R^{n}}{r}
\end{gathered}
$$

and

$$
\int_{Q_{L}} \zeta \cdot \mu|\nabla u| \gtrsim R^{n}
$$

We first show how (5.7), (5.8) and (5.9) imply

$$
\sup _{B_{\sqrt{n} R}(a) \cap Q_{L}}|v| \lesssim \frac{1}{r^{2}}+\frac{1}{r} R
$$

for all $r, R$ satisfying (5.6). Note that since $a$ can be any point such that $B_{R}(a) \subset$ $Q_{L}$, inequality (5.10) will imply an upper bound on $v\left(x_{0}\right)$ which holds for all $x_{0} \in$ $Q_{L}$, and thus we have (1.16). For $x_{0} \in B_{\sqrt{n} R}(a) \cap Q_{L}$, we have

$$
\begin{aligned}
v\left(x_{0}\right) \int_{Q_{L}} \zeta \cdot \mu|\nabla u| & =\int_{Q_{L}} v \zeta \cdot \mu|\nabla u|+\int_{Q_{L}}\left(v\left(x_{0}\right)-v\right) \zeta \cdot \mu|\nabla u| \\
& =-\int_{Q_{L}}(\operatorname{div} \zeta-\mu \cdot(D \zeta \mu))|\nabla u|+\int_{Q_{L}}\left(v\left(x_{0}\right)-v\right) \zeta \cdot \mu|\nabla u|,
\end{aligned}
$$


and hence

$$
\begin{aligned}
& \left|v\left(x_{0}\right)\right| \underset{[\sqrt{5.9}}{\lesssim} \frac{1}{R^{n}}\left(\left|\int_{Q_{L}}(\operatorname{div} \zeta-\mu \cdot(D \zeta \mu))\right| \nabla u||+\left|\int_{Q_{L}}\left(v\left(x_{0}\right)-v\right) \zeta \cdot \mu\right| \nabla u \mid\right) \\
& \underset{[5.7,[5.8]}{\lesssim} \frac{1}{R^{n}}\left(\frac{R^{n}}{r^{2}}+\sup _{B_{R}(a)}\left|v-v\left(x_{0}\right)\right| \frac{R^{n}}{r}\right) \\
& \underset{(5.5)}{\lesssim} \frac{1}{r^{2}}+\frac{R}{r}
\end{aligned}
$$

which gives (5.10).

It remains to prove (5.7), (5.8) and (5.9). We start with (5.7). First notice that

$$
\sup _{B_{R / 2}(a)}\left|\nabla u_{r r}^{* *}\right| \leq \sup _{B_{R / 2+r / 4}(a)}\left|\nabla u_{r}^{*}\right| \leq \sup _{B_{R / 2+r / 2}(a)}|u| \int_{\mathbb{R}^{n}}\left|\nabla \phi_{r}\right| \lesssim \frac{1}{r}
$$

and

$$
\sup _{B_{R / 2}(a)}\left|D^{2} u_{r r}^{* *}\right| \leq \sup _{B_{R / 2+r / 4}(a)}\left|D^{2} u_{r}^{*}\right| \leq \sup _{B_{R / 2+r / 2}(a)}|u| \int_{\mathbb{R}^{n}}\left|D^{2} \phi_{r}\right| \lesssim \frac{1}{r^{2}} .
$$

Thus from

$$
D \zeta=\eta_{R} D^{2} u_{r r}^{* *}+\nabla u_{r r}^{* *} \otimes \nabla \eta_{R}
$$

we obtain

$$
\sup _{\mathbb{R}^{n}}|D \zeta| \lesssim \sup _{B_{R / 2}(a)}\left|D^{2} u_{r r}^{* *}\right|+\frac{1}{R} \sup _{B_{R / 2}(a)}\left|\nabla u_{r r}^{* *}\right| \lesssim \frac{1}{r^{2}}+\frac{1}{r R} \underset{(5.6)}{\lesssim} \frac{1}{r^{2}}
$$

Thus

$$
\left|\int_{Q_{L}}(\operatorname{div} \zeta-\mu \cdot(D \zeta \mu))\right| \nabla u|| \leq \sup _{\mathbb{R}^{n}}|D \zeta| \int_{B_{R / 2}(a)}|\nabla u| \underset{(5.4), \underset{(5.13)}{\lesssim}}{\lesssim} \frac{R^{n}}{r^{2}},
$$

which gives (5.7).

Estimate (5.8) is very similar. Indeed,

$$
\sup _{B_{R}(a)}|\zeta| \leq \sup _{B_{R / 2}(a)}\left|\nabla u_{r r}^{* *}\right| \underset{(5.11)}{\lesssim} \frac{1}{r}
$$

and hence

$$
\int_{Q_{L}}|\zeta \cdot \mu||\nabla u| \leq \sup _{\mathbb{R}^{n}}|\zeta| \int_{B_{R / 2}(a)}|\nabla u| \underset{(5.4),(5.14)}{\lesssim} \frac{R^{n}}{r} .
$$

The proof of (5.9) is more involved. In order to focus on the heart of the estimate, note that (5.9) follows from the next two estimates if $R$ is chosen sufficiently large, that is for a suitable choice of constant $C_{1}$ in (5.6): There exists a constant $C$ such that

$$
\left.\left|\int_{Q_{L}} \zeta \cdot \mu\right| \nabla u\left|-\int_{Q_{L}} \eta_{R}\right| \nabla u_{r}^{*}\right|^{2} \mid \leq C R^{n-1}
$$

and

$$
\int_{Q_{L}} \eta_{R}\left|\nabla u_{r}^{*}\right|^{2} \geq \frac{1}{C} R^{n}
$$


To prove (5.15), note that

$$
\begin{aligned}
\int_{Q_{L}} \zeta \cdot \mu|\nabla u|-\int_{Q_{L}} \eta_{R}\left|\nabla u_{r}^{*}\right|^{2} & =\int_{Q_{L}}\left(\eta_{R} \nabla u_{r r}^{* *}\right) \cdot \mu|\nabla u|-\int_{Q_{L}}\left(\eta_{R} \nabla u_{r}^{*}\right)_{r}^{*} \cdot \nabla u \\
& =\int_{Q_{L}}\left(\eta_{R} \nabla u_{r r}^{* *}-\left(\eta_{R} \nabla u_{r}^{*}\right)_{r}^{*}\right) \cdot \mu|\nabla u| .
\end{aligned}
$$

Hence

$$
\begin{aligned}
\left.\left|\int_{Q_{L}} \zeta \cdot \mu\right| \nabla u\left|-\int_{Q_{L}} \eta_{R}\right| \nabla u_{r}^{*}\right|^{2} \mid & \leq \sup _{B_{R}(a)}\left|\eta_{R} \nabla u_{r r}^{* *}-\left(\eta_{R} \nabla u_{r}^{*}\right)_{r}^{*}\right| \int_{B_{R / 2}(a)}|\nabla u| \\
& \underset{\mathbf{5 . 5 . 4}}{\lesssim} R^{n} \sup _{B_{R}(a)}\left|\eta_{R} \nabla u_{r r}^{* *}-\left(\eta_{R} \nabla u_{r}^{*}\right)_{r}^{*}\right| .
\end{aligned}
$$

For the supremum note that

$$
\left(\eta_{R} \nabla u_{r r}^{* *}-\left(\eta_{R} \nabla u_{r}^{*}\right)_{r}^{*}\right)(x)=\int_{\mathbb{R}^{n}}\left(\eta_{R}(x)-\eta_{R}(x-y)\right) \nabla u_{r}^{*}(x-y) \phi_{r}(y) d y,
$$

so that

$$
\begin{aligned}
\sup _{B_{R}(a)}\left|\eta_{R} \nabla u_{r r}^{* *}-\left(\eta_{R} \nabla u_{r}^{*}\right)_{r}^{*}\right| & \leq \sup _{B_{R}(a)}\left|\nabla \eta_{R}\right| \sup _{B_{R / 2+r / 4}}\left|\nabla u_{r}^{*}\right| \int_{\mathbb{R}^{n}}|y| \phi_{r}(y) d y \\
& \underset{[5.11]}{\lesssim} \frac{1}{R} \frac{1}{r} r=\frac{1}{R} .
\end{aligned}
$$

The combination of (5.17) and (5.18) yields (5.15).

It remains to prove (5.16), which is the most involved. First we note that (5.16) will follow if we can show

$$
\int_{B_{h}(\tilde{a})}\left|\nabla u_{r}^{*}\right|^{2} \geq \frac{1}{C} h^{n-2}
$$

for all $\tilde{a}, r, h$ with $B_{2 h}(\tilde{a}) \subset Q_{L}$ and

$$
r \leq \frac{1}{C_{1}} \leq C_{2} \leq h
$$

where $C_{2} \geq 1$, and hence $R$, are now chosen such that $R \geq 4 C_{2} \geq C_{1}$. To see this, let $h=C_{2}$. There exist a family of points $\left\{\tilde{a}_{i}\right\}_{i \in I}$ such that

$$
B_{h}\left(\tilde{a}_{i}\right) \subset B_{R / 4}(a), \quad\left\{B_{h}\left(\tilde{a}_{i}\right)\right\}_{i \in I} \text { are disjoint, } \quad \# I \geq \frac{1}{C} \frac{R^{n}}{h^{n}} .
$$

Hence we have

$$
\begin{aligned}
\int_{Q_{L}} \eta_{R}\left|\nabla u_{r}^{*}\right|^{2} & \geq \quad \int_{B_{R / 4}(a)}\left|\nabla u_{r}^{*}\right|^{2} \\
& \geq \quad \sum_{i \in I} \int_{B_{h}\left(\tilde{a}_{i}\right)}\left|\nabla u_{r}^{*}\right|^{2} \\
\underset{(5.20)}{\geq} & \frac{1}{C} \frac{R^{n}}{h^{n}} h^{n-2}=\frac{1}{C} \frac{R^{n}}{h^{2}}=\frac{1}{C C_{2}^{2}} R^{n} .
\end{aligned}
$$


We turn to establishing (5.19). It will follow from the following four estimates:

$$
\begin{gathered}
\int_{B_{h}(\tilde{a})}\left|u_{r}^{*}-\frac{\int \eta_{h} u_{r}^{*}}{\int \eta_{h}}\right|^{2} \leq C h^{2} \int_{B_{h}(\tilde{a})}\left|\nabla u_{r}^{*}\right|^{2}, \\
\left|\int_{B_{h}(\tilde{a})} \eta_{h}\left(u_{r}^{*}-m\right)\right| \\
\int_{B_{h}(\tilde{a})}\left|u_{r}^{*}-u\right|^{2} \leq C r h_{B_{h}(\tilde{a})} \eta_{h}, \\
\int_{B_{h}(\tilde{a})}|u-m|^{2} \geq \frac{1}{C} h^{n}
\end{gathered}
$$

for some constant $C$. To see this, note that

$$
\begin{aligned}
& h^{n} \underset{[5.24)}{\leq} \quad C \int_{B_{h}(\tilde{a})}|u-m|^{2} \\
& \leq \quad C\left(\int_{B_{h}(\tilde{a})}\left|u_{r}^{*}-m\right|^{2}+\int_{B_{h}(\tilde{a})}\left|u-u_{r}^{*}\right|^{2}\right) \\
& \underset{(5.23)}{\leq} \quad C \int_{B_{h}(\tilde{a})}\left|u_{r}^{*}-m\right|^{2}+C r h^{n} \\
& \leq \quad C\left(\int_{B_{h}(\tilde{a})}\left|u_{r}^{*}-\frac{\int \eta_{h} u_{r}^{*}}{\int \eta_{h}}\right|^{2}+h^{n}\left|\frac{\int \eta_{h}\left(u_{r}^{*}-m\right)}{\int \eta_{h}}\right|^{2}\right)+C r h^{n} \\
& \underset{(5.21),(5.22)}{\leq} C\left(h^{2} \int_{B_{h}(\tilde{a})}\left|\nabla u_{r}^{*}\right|^{2}+h^{n-2}+r h^{n}\right),
\end{aligned}
$$

and so

$$
\int_{B_{h}(\tilde{a})}\left|\nabla u_{r}^{*}\right|^{2} \geq \frac{1}{C} h^{n-2}-h^{n-4}-r h^{n-2} .
$$

We lastly address (5.21) - (5.24). Upon rescaling, (5.21) turns into

$$
\int_{B_{1}(0)}\left|\tilde{u}-\frac{\int \eta_{1} \tilde{u}}{\int \eta_{1}}\right|^{2} \lesssim \int_{B_{1}(0)}|\nabla \tilde{u}|^{2}
$$

where $\tilde{u}$ is a re-scaled version of $u_{r}^{*}$. This inequality is a version of Poincaré's inequality with mean value zero and is established via a compactness argument.

Estimate (5.22) follows since

$$
\int_{B_{h}(\tilde{a})} \eta_{h}\left(u_{r}^{*}-m\right)=\int_{B_{h}(\tilde{a})} \eta_{h}\left(-\triangle v_{r}^{*}\right)=\int_{B_{h}(\tilde{a})} \nabla \eta_{h} \cdot \nabla v_{r}^{*}
$$

implies

$$
\left|\int_{B_{h}(\tilde{a})} \eta_{h}\left(u_{r}^{*}-m\right)\right| \leq \sup |\nabla v| \int_{B_{h}(\tilde{a})}\left|\nabla \eta_{h}\right| \underset{\substack{5.5 \\[5}}{\lesssim} h^{n-1}=\frac{1}{h} \int_{B_{h}(\tilde{a})} \eta_{h} .
$$

For estimate (5.23), note that since $u_{r}^{*}-u$ is bounded,

$$
\int_{B_{h}(\tilde{a})}\left|u_{r}^{*}-u\right|^{2} \lesssim \int_{B_{h}(\tilde{a})}\left|u_{r}^{*}-u\right|
$$


We also have

$$
\int_{B_{h+r / 4}(\tilde{a})}|\nabla u| \underset{(5.4)}{\lesssim}(h+r / 4)^{n} \lesssim h^{n}
$$

Thus the estimate (5.23) nows follows from the standard estimate

$$
\int_{B_{h}(\tilde{a})}\left|u_{r}^{*}-u\right| \lesssim r \int_{B_{h+r / 4}(\tilde{a})}|\nabla u| .
$$

Finally (5.24) follows from the fact that $u \in\{-1,1\}$ and $m \in(-1,1)$.

\section{Interpretation And CONSEquences of Theorem 1.3}

Recall from the Introduction the notion of large scale average volume fraction fluctuations: That is, for $\phi_{R}$ satisfying (1.11) and (1.12), we take any admissible $(u, \mathbf{b})$ on $\mathcal{A}_{0}\left(Q_{L}\right)$ for a very large value of $L$, and consider the quantity

$$
\int_{B_{R}(a)} \phi_{R}(x)(u(x)-m) d x
$$

for $B_{R}(a) \subset Q_{L}$. We are concerned with the rate of decay of (6.1) as $R$ gets larger. As we described in the Introduction, the bound on the potential yields a certain rate of decay of these fluctuations as $R$ tends to $+\infty$. Our goal here is use Theorem 1.3 to show that, while this rate of decay is much weaker than rates for a truly periodic structure, for $n<4$, it is stronger than the one for a random checkerboard pattern. In this sense, the optimal pattern has less large scale variations of the average volume fraction than a pattern with a finite correlation length.

In Proposition 6.1, we investigate the behaviour of quantity (6.1) in several cases, to conclude that, while our result (6.6) for a minimizer is much weaker than for the periodic structure (6.5), for $n<4$, it is stronger than the behavior of a generic random pattern with strongly decaying correlations. Let us first make a few definitions.

By a genuinely periodic structure $u$, we mean a function $u$ defined on $\mathbb{R}^{n}$ such that for some $l_{*}$, we have

$$
u(x):=\hat{u}\left(\frac{x}{l_{*}}\right),
$$

where $\hat{u}$ is 1 -periodic with mean $m$, i.e.,

$$
\hat{u}(x+k)=\hat{u}(x) \quad \forall x \in \mathbb{R}^{n}, k \in \mathbb{Z}^{n} \quad \text { and } \quad \int_{Q_{1}} \hat{u} d x=m .
$$

We define what we mean by a generic random pattern with strongly decaying correlations. For convenience, we define our pattern over $\mathbb{R}^{n}$. For each $x \in \mathbb{R}^{n}$, let $u^{*}(x)$ be a random variable with mean $m$. We use $\left\langle u^{*}(x)\right\rangle$ to denote the expected value (ensemble average). We further assume that the spatial covariance function $\mathcal{V}$ of the random field, $u^{*}(x), x \in \mathbb{R}^{n}$, decays on a length scale $l_{*}$ in the sense that

$$
\left\langle\left(u^{*}(x)-m\right)\left(u^{*}(y)-m\right)\right\rangle=\mathcal{V}\left(\frac{x-y}{l_{*}}\right) \quad \forall x, y, \in \mathbb{R}^{n},
$$

with

$$
\int_{\mathbb{R}^{n}}|\mathcal{V}(\cdot)|<\infty
$$


We will use the term generic (cf. Remark 6.2) to mean

$$
\int_{\mathbb{R}^{n}} \mathcal{V}(\cdot) \neq 0
$$

Proposition 6.1. We have the following:

(i) If $u$ is a genuinely periodic structure, then for any $a \in \mathbb{R}^{n}$ and positive integer $p$ there holds

$$
\left|\int_{B_{R}(a)} \phi_{R}(x)(u(x)-m) d x\right| \leq C(p, u) \frac{1}{R^{2 p}} .
$$

(ii) If $\left(u_{0}, \mathbf{b}_{0}\right)$ is a minimizer of $E\left(u, \mathbf{b}, Q_{L}\right)$ over $\mathcal{A}_{0}$, then

$$
\left|\int_{B_{R}(a)} \phi_{R}(x)\left(u_{0}(x)-m\right) d x\right| \leq C(n, m) \frac{1}{R^{2}} .
$$

(iii) If $u^{*}$ is a generic random configuration with mean $m$ and strongly decaying correlations, then

$$
\left\langle\left(\int_{B_{R}(a)} \phi_{R}(x)\left(u^{*}(x)-m\right) d x\right)^{2}\right\rangle^{1 / 2} \geq C\left(u^{*}\right) \frac{1}{R^{n / 2}} .
$$

Proof. (i) Fix $p \in \mathbb{N}$. Since $\hat{u}-m$ is periodic with mean zero on $Q_{1}$, there exists a bounded periodic $\hat{v}$ on $\mathbb{R}^{n}$ such that

$$
\triangle^{p} \hat{v}=\hat{u}-m .
$$

Hence

$$
\begin{aligned}
\left|\int_{\mathbb{R}^{n}} \phi_{R}(x)(u(x)-m) d x\right| & =\left|\int_{\mathbb{R}^{n}} \phi_{R}(x) \triangle_{y}^{p} \hat{v}\left(\frac{x}{l_{*}}\right) d x\right| \\
& =\left|l_{*}^{2 p} \int_{\mathbb{R}^{n}} \triangle_{x}^{p} \phi_{R}(x) v\left(\frac{x}{l_{*}}\right) d x\right| \\
& \leq l_{*}^{2 p} \sup _{\mathbb{R}^{n}}|v| \int_{\mathbb{R}^{n}}\left|\triangle_{x}^{p} \phi_{R}(x)\right| d x \\
& \leq C\left(p, l_{*}\right) \frac{1}{R^{2 p}} .
\end{aligned}
$$

(ii) This follows directly from Theorem 1.3 by integrating by parts once more in (1.15).

(iii) We claim that

$$
\lim _{R \rightarrow \infty} R^{n}\left\langle\left(\int_{\mathbb{R}^{n}} \phi_{R}\left(u^{*}-m\right)\right)^{2}\right\rangle=\left(\int_{\mathbb{R}^{n}} \phi_{1}^{2}\right)\left(\int_{\mathbb{R}^{n}} \mathcal{V}(\cdot)\right) l_{*}^{n} .
$$

Hence by (6.4) we have

$$
\left\langle\left(\int_{\mathbb{R}^{n}} \phi_{R}\left(u^{*}-m\right)\right)^{2}\right\rangle \geq \frac{1}{C(\mathcal{V})} \frac{1}{R^{n / 2}} l_{*}^{n / 2}
$$

for $R$ sufficiently large. 
To show (6.8), we note that

$$
\begin{aligned}
\left\langle\left(\int \phi_{R}(u-m)\right)^{2}\right\rangle & =\left\langle\iint \phi_{R}(x) \phi_{R}(y)\left(u^{*}(x)-m\right)\left(u^{*}(y)-m\right) d x d y\right\rangle \\
& =\iint \phi_{R}(x) \phi_{R}(y)\left\langle\left(u^{*}(x)-m\right)\left(u^{*}(y)-m\right)\right\rangle d x d y \\
& =\iint \phi_{R}(x) \phi_{R}(y) \mathcal{V}\left(\frac{x-y}{l_{*}}\right) d x d y \\
& =l_{*}^{n} \iint \phi_{R}(x) \phi_{R}\left(x-l_{*} \hat{z}\right) d x \mathcal{V}(\hat{z}) d \hat{z} \\
& =\frac{l_{*}^{n}}{R^{n}} \iint \phi_{1}(\hat{x}) \phi_{1}\left(\hat{x}-l_{*} R^{-1} \hat{z}\right) d \hat{x} \mathcal{V}(\hat{z}) d \hat{z} .
\end{aligned}
$$

Since

$$
\lim _{R \rightarrow \infty} \int \phi_{1}(\hat{x}) \phi_{1}\left(\hat{x}-l_{*} R^{-1} \hat{z}\right) d \hat{x}=\int \phi_{1}^{2}(\hat{x}) d \hat{x}
$$

equation (6.8) follows from (6.10), (6.3) and Lebesgue dominated convergence theorem.

Remark 6.2. We end by noting that (6.2), (6.3) and (6.4) hold for a random checkerboard pattern. By this we mean a function $u^{*}$ which is piecewise constant on the cubes

$$
\left\{h+k l_{*}+\left(0, l_{*}\right)^{n}\right\}_{k \in \mathbb{Z}^{n}}
$$

for some fixed $h \in\left(0, l_{*}\right)^{n}$, and such that the values on these cubes are independent, identically distributed random variables taking the value 1 with probability $(1+$ $m) / 2$ and the value -1 with probability $(1-m) / 2$. For fixed $h \in\left(0, l_{*}\right)^{n}$, we have

$$
\left\langle\left(u^{*}(x)-m\right)\left(u^{*}(y)-m\right)\right\rangle=\left\{\begin{array}{cl}
1-m^{2} & \text { if } x, y \text { are in the same cube } \\
0 & \text { otherwise }
\end{array}\right.
$$

In order to make the pattern statistically homogeneous, we randomize the shift $h$ to make it uniformly distributed over $\left(0, l_{*}\right)^{n}$. We obtain

$$
\begin{aligned}
\left\langle\left(u^{*}(x)-m\right)\left(u^{*}(y)-m\right)\right\rangle= & \left(1-m^{2}\right) p(x, y \text { are in the same cube }) \\
= & \left(1-m^{2}\right) \max \left\{1-\frac{\left|x_{1}-y_{1}\right|}{l_{*}}, 0\right\} \\
& \cdots \max \left\{1-\frac{\left|x_{n}-y_{n}\right|}{l_{*}}, 0\right\} .
\end{aligned}
$$

The covariance function $\mathcal{V}$ is hence given by

$$
\mathcal{V}(z)=\left(1-m^{2}\right) \max \left\{1-\frac{\left|z_{1}\right|}{l_{*}}, 0\right\} \cdots \max \left\{1-\frac{\left|z_{n}\right|}{l_{*}}, 0\right\}
$$

which clearly satisfies (6.2), (6.3) and (6.4). 


\section{ACKNOWLEDGMENTS}

The research of the first author was partially supported by the MURST research project Calcolo delle Variazioni. The research of the second author was partially supported by an NSERC (Canada) Discovery Grant. The research of the third author was partially supported by the German Science Foundation through SFB 611 and by the Hausdorff Center for Mathematics. The third author also thanks the Pacific Institute of Mathematical Sciences for their hospitality. We would also like to thank the anonymous referees for many helpful comments on the manuscript.

\section{REFERENCES}

[1] Alberti, G.; Müller, S.: A new approach to variational problems with multiple scales. Comm. Pure Appl. Math. 54 (2001), no. 7, 761-825. MR1823420 (2002f:49016)

[2] Bates, F.S.; Fredrickson, G.H.: Block copolymers - Designer soft materials. Physics Today 52 (1999), no. 2, 32-38.

[3] Brézis, H.: Opèrateurs maximaux monotones et semi-groupes de contractions dans les espaces de Hilbert. North-Holland Mathematics Studies 5. North-Holland Publishing Co., Amsterdam, 1973. MR0348562 (50:1060)

[4] Chen, X.; Oshita, Y.: Periodicity and uniqueness of global minimizers of an energy functional containing a long-range interaction. SIAM J. Math. Anal. 37 (2005), no. 4, 1299-1332. MR2192296 (2006k:49027)

[5] Chen, X.; Oshita, Y.: An application of the modular function in nonlocal variational problems. Arch. Ration. Mech. Anal. 186 (2007), no. 1, 109-132. MR2338353

[6] Choksi, R.: Scaling laws in microphase separation of diblock copolymers. J. Nonlinear Sci. 11 (2001), no. 3, 223-236. MR1852942 (2003h:82091)

[7] Choksi, R.; Kohn, R.V.; Otto, F.: Domain branching in uniaxial ferromagnets: a scaling law for the minimum energy. Comm. Math. Phys. 201 (1999), no. 1, 61-79. MR1669433 (2000c:49060)

[8] Choksi, R.; Peletier, M.A.; Williams, J.F.: On the phase diagram for microphase separation of diblock copolymers: an approach via a nonlocal Cahn-Hilliard functional. Submitted.

[9] Choksi, R.; Ren, X.: On the derivation of a density functional theory for microphase separation of diblock copolymers. J. Statist. Phys. 113 (2003), no. 1-2, 151-176. MR2012976 (2004k:82065)

[10] Choksi, R.; Sternberg, P.: On the first and second variations of a nonlocal isoperimetric problem. J. Reine Angew. Math. 611 (2007), 75-108. MR2360604

[11] Conti, S.: Branched microstructures: scaling and asymptotic self-similarity. Comm. Pure Appl. Math. 53 (2000), no. 11, 1448-1474. MR.1773416 (2001j:74032)

[12] DeSimone, A.; Kohn, R.V.; Müller, S.; Otto, F.: Recent analytical developments in micromagnetics. In The science of hysteresis. Vol. II. Physical modeling, micromagnetics, and magnetization dynamics (G. Bertotti and I.D. Mayergoyz. eds.), pp. 269-381. Elsevier/Academic Press, Amsterdam, 2006. MR2307930

[13] Giusti, E.: Minimal surfaces and functions of bounded variation. Monographs in Mathematics, 80. Birkhäuser Verlag, Basel, 1984. MR775682 (87a:58041)

[14] Goldstein, R.E.; Muraki, D.J.; Petrich, D.M.: Interface proliferation and the growth of labyrinths in a reaction-diffusion system. Phys. Rev. E (3) $\mathbf{5 3}$ (1996), no. 4, part B, 39333957. MR:1388238 (97b:35100)

[15] Kohn, R.V.: Energy-driven pattern formation. In International Congress of Mathematicians. Proceedings of the Congress held in Madrid, August 22-30, 2006. (M. Sanz-Solž et al., eds.), vol. I, pp. 359-383. European Mathematical Society (EMS), Zürich, 2007. MR2334197

[16] Lieb, E.; Loss, M.: Analysis. Graduate Studies in Mathematics, 14. American Mathematical Society, Providence, RI, 1997. MR1415616 (98b:00004)

[17] Müller, S.: Singular perturbations as a selection criterion for periodic minimizing sequences. Calc. Var. Partial Differential Equations 1 (1993), no. 2, 169-204. MR.1261722 (95k:49030)

[18] Muratov, C.B.: Theory of domain patterns in systems with long-range interactions of Coulomb type. Phys. Rev. E (3) 66 (2002), no. 6, 066108. MR.1953930 (2003k:82028) 
[19] Nishiura, Y.; Ohnishi, I.: Some mathematical aspects of the micro-phase separation in diblock copolymers. Physica D 84 (1995), no. 1-2, 31-39. MR1334695 (96g:35196)

[20] Ohnishi, I.; Nishiura, Y.; Imai, M.; Matsushita, Y.: Analytical solutions describing the phase separation driven by a free energy functional containing a long-range interaction term. Chaos 9 (1999), no. 2, 329-341. MR1697656 (2000d:35244)

[21] Ohta, T.; Kawasaki, K.: Equilibrium morphology of block copolymer melts. Macromolecules 19 (1986), no. 10, 2621-2632.

[22] Ren, X.; Wei, J.: On energy minimizers of the diblock copolymer problem. Interfaces Free Bound. 5 (2003), no. 2, 193-238. MR.1980472 (2004i:82077)

[23] Ren, X.; Wei, J.: Wriggled lamellar solutions and their stability in the diblock copolymer problem. SIAM J. Math. Anal. 37 (2005), no. 2, 455-489. MR2176111 (2006m:35112)

[24] Ren, X.; Wei, J.: Existence and stability of spherically layered solutions of the diblock copolymer equation. SIAM J. Appl. Math. 66 (2006), no. 3, 1080-1099. MR2216732 (2007a:34088)

[25] Seul, M.; Andelman, D.: Domain shapes and patterns: The phenomenology of modulated phases. Science 267 (1995), no. 5197, 476-483.

[26] Teramoto, T.; Nishiura, Y.: Double gyroid morphology in a gradient system with nonlocal effects. Journal of the Physical Society of Japan 71 (2002), no. 7, 1611-1614.

[27] Theil, F.: A proof of crystallization in two dimensions. Comm. Math. Phys. 262 (2006), no. 1, 209-236. MR2200888 (2007f:82018)

[28] Thomas, E.L; Anderson, D.M.; Henkee, C.S.; Hoffman, D.: Periodic area-minimizing surfaces in block copolymers. Nature 334 (1988), no. 6183, 598-601.

[29] Yip, N.K.: Structure of stable solutions of a one-dimensional variational problem. ESAIM Control Optim. Calc. Var. 12 (2006), no. 4, 721-751. MR2266815 (2007g:49027)

Dipartimento di Matematica, Università di Pisa, largo Pontecorvo 5, 56127 Pisa, ITALY

E-mail address: galberti1@dm.unipi.it

Department of Mathematics, Simon Fraser University, 8888 University Drive, BurnABY, BC V5A 1S6 CANADA

E-mail address: choksi@math.sfu.ca

Institute for Applied Mathematics, Universität Bonn, Wegelerstr 10, D-53115 Bonn, GERMANY

E-mail address: otto@iam.uni-bonn.de 